\title{
Are digital technology interventions effective to reduce loneliness in older adults? A systematic review and meta-analysis
}

Syed Ghulam Sarwar Shah MBBS, MA, MSc, $\mathrm{PhD}^{1,2, *}$, David Nogueras MBA ${ }^{3}$, Hugo Cornelis van Woerden MBChB, $\mathrm{PhD}^{4,5}$, Vasiliki Kiparoglou MSc, MBA, PhD ${ }^{1,6}$

${ }^{1}$ NIHR Oxford Biomedical Research Centre, Oxford University Hospitals NHS Foundation Trust, John Radcliffe Hospital, Headington, Oxford, OX3 9DU, England, UK

${ }^{2}$ Radcliffe Department of Medicine, University of Oxford, Level 6, West Wing, John Radcliffe Hospital, Headington, Oxford OX3 9DU, England, UK

${ }^{3}$ EvZein Limited, Holley Crescent, Headington, Oxford, OX38AW, England, UK

${ }^{4}$ Public Health Agency, 12 - 22 Linenhall Street, Belfast, BT2 8BS, Northern Ireland, UK

${ }^{5}$ Centre for Health and Science, University of the Highlands and Islands, Old Perth Road, Inverness, IV2 3JH, Scotland, UK

${ }^{6}$ Nuffield Department of Primary Care Health Sciences, University of Oxford, Radcliffe Observatory Quarter, Woodstock Road, Oxford. OX2 6GG, England, UK

${ }^{*}$ Corresponding author

Email: Sarwar.Shah@ouh.nhs.uk 


\title{
Are digital technology interventions effective to reduce loneliness in older adults: A systematic review and meta- analysis
}

\author{
ABSTRACT: \\ Objective: To review the latest literature on the effectiveness of DTIs in reducing loneliness \\ in (older) adults.
}

Data Sources: Electronic searches in PubMed, Medline, CINAHL, EMBASE and Web of Science covering publication period from 1 January 2010 to 31 July 2019.

Subjects: Adult men and women

Design: Systematic review and meta-analysis

Main Outcome Measure: Loneliness.

Study Selection: Primary studies that used DTIs for tackling loneliness in adults (aged $\geq 18$ years) with follow-up measurements at least three months or more and publication in the English language.

Data Extraction and Synthesis: Two researchers independently screened articles and extracted data on several variables: participants, interventions, comparators and outcomes. Data was extracted on the primary outcome i.e. loneliness measured at the baseline and follow-up measurements at three, four, six and twelve months after the intervention.

Results: Six studies were selected from 4939 articles screened. Selected studies included 5 clinical trials (4 RCTs and 1 quasi experimental study) and one before and after study, which enrolled 646 participants $($ men $=154(24 \%)$, women $=427(66 \%)$, no gender information $=65(10 \%)$ with average age between 73 and 78 years (SD 6-11). Five clinical trials were included in the meta-analysis and standardised mean differences (SMD) were calculated for each trial and pooled across studies using a random effects model. The overall effect estimates were not statistically significant in follow-up measurements at three months $(\mathrm{SMD}=0.02,95 \% \mathrm{Cl}=-0.36,0.40 ; \mathrm{P}=0.92)$, four months $(\mathrm{SMDs}=-1.11,95 \% \mathrm{Cl}=-$ $2.60,0.38 ; \mathrm{P}=0.14)$ and six months $(\mathrm{SMD}=-0.11,95 \% \mathrm{Cl}=-0.54,0.32 ; \mathrm{P}=0.61)$. The quality of evidence was very low to moderate in these trials.

Conclusions: There is insufficient evidence to make conclusions that DTls are effective in reducing loneliness in older adults. Future research may consider RCTs with larger sample sizes and longer duration of interventions and follow-up.

\section{INTRODUCTION}

Loneliness is an important public health problem (1), which has been seriously exacerbated because of recent isolation, social distancing, and lockdown measures for tackling the COVID-19 pandemic $(2,3)$. Thus, the burden of loneliness is expected to rise due to the COVID-19 crisis in countries (4) beyond developed countries where it is already high (5-12). The use of digital technology intervention have been suggested for mitigating the impacts of 
medRxiv preprint doi: https://doi.org/10.1101/2020.08.27.20183012; this version posted September 1,2020 . The copyright holder for this preprint (which was not certified by peer review) is the author/funder, who has granted medRxiv a license to display the preprint in

It is made available under a CC-BY 4.0 International license .

loneliness and attempting to deal with isolation due to social distancing in the epidemic situations such as the COVID-19 pandemic (3).

Loneliness refers to subjective feelings of an individual because of a perceived discrepancy between actual and the desired social relationships $(13,14)$. While loneliness affects people of all ages, $(14,15)$ older and vulnerable people are affected more $(10,16,17)$. It is associated with the social and physical environment (16-18). Loneliness enhances the risk of poor mental and physical health $(13,19-22)$, dementia (23), premature mortality and allcause mortality (20) particularly in older adults (22).

Loneliness could be addressed through a range of social (24) and technological interventions (25) such as digital applications (apps), online social networks, and social robots (26).

However, there is limited evidence on the effectiveness of technological interventions for loneliness (27). While several previously published reviews report that these interventions are effective in reducing loneliness (28-32), some of these studies are weak and have a high risk of bias (33). Some use very selected technological interventions and cover literature published over a very short time period i.e. between January 2010 and January 2013 (29). These findings suggest the need for further research $(30,31)$ to assess and identify the latest digital technological interventions that are effective in reducing loneliness $(27,32)$.

We therefore appraise the latest evidence to assess the effectiveness of digital technology interventions to reduce loneliness, which is imperative not only from the perspectives of patients and their families but also from the perspectives of other stakeholders such as public health, health and social care providers, and health insurers (34).

\subsection{Study objectives}

Our main objective was to assess the effectiveness of digital technology interventions (DTIs) to reduce loneliness in adults. The secondary objective was to identify DTIs that are used to reduce loneliness in adults.

\subsection{Review questions:}

Our primary question was: "Are DTIs effective for reducing loneliness in adults?" and the secondary question was "What DTls are used for reducing loneliness in adults?"

\subsection{Outcome measures:}

Our main outcome measure was loneliness. We extracted loneliness scores measured at the baseline (before the intervention) and the follow-up measurements (at the end of intervention or sometime after the intervention) for the intervention group, and the control group(s) if any. 
medRxiv preprint doi: https://doi.org/10.1101/2020.08.27.20183012; this version posted September 1, 2020. The copyright holder for this preprint (which was not certified by peer review) is the author/funder, who has granted medRxiv a license to display the preprint in perpetuity.

It is made available under a CC-BY 4.0 International license .

\section{METHODS}

\subsection{Protocol registration and publication}

We registered our systematic review and meta-analysis with the PROSPERO database on $10^{\text {th }}$ June 2019 (Registration ID CRD42019131524) (35) and we published our protocol (34).

\subsection{Definition: Digital technology intervention}

We defined a digital technology intervention as an intervention that applies digital technology i.e. the technology, equipment and applications that process information in the form of numeric codes, usually a binary code (36).

\subsection{Eligibility Criteria}

We selected studies that met our predefined eligibility Criteria (34). These included interventional studies (randomised and non-randomised) on loneliness using DTIs. The DTIs included the use of computers, computer tablets, iPads, Internet, online videos, communication, chatting, social groups, meetings, conferences and messages, sensors, (social) robots, (smart) phones, social media tools and the World Wide Web. A minimum Intervention duration and follow-up period was set at three months. Participants included both male and female adults (aged 18 years and more) living in different settings (i.e. residential dwellings including private residences and care / nursing homes in any country. The studies were limited to journal articles in the English language published from 1 January 2010 to 31 July 2019.

\subsection{Information sources and keywords}

We did electronic searches in PubMed, Medline, EMBASE, CINAHL and Web of Science covering the publication period from 1 January 2010 to 31 July 2019. We used an a priori list of keywords prepared in our preliminary literature searches (34). The keywords were of two categories: medical condition / problem (i.e. loneliness, lonely, isolation, aloneness, disconnect ${ }^{*}$, solitude, singleness ${ }^{*}$, lonesomeness, solitariness, and remoteness).and intervention / technology (i.e. digital, technolog ${ }^{*}$, sensor ${ }^{*}$, robot $^{*}$, internet, social media, *phone*, online, iPad ${ }^{*}$, tablet ${ }^{*}$, computer ${ }^{*}$, electronic, web, video, and videoconference), as reported in our protocol (34).

\subsection{Literature searches}

First, we searched the keywords in the 'subject headings' such as Medical Subject Headings (MeSH) major terms in the PubMed or equivalent in other databases (Appendix-1). Thereafter, we searched for keywords in the 'title' and 'abstract' fields in the selected databases using three Boolean operators: 'OR', 'AND' and 'NOT'. In addition, we hand searched through reference lists of shortlisted articles. We wrote emails to the authors of two studies for full copies of their research articles $(37,38)$, which were gratefully provided to us. We contacted the authors of two further studies for missing / additional data $(39,40)$. We had good response from the authors of both studies and data were thankfully provided for one study (40). We sought support from an expert librarian for running literature searches. 
medRxiv preprint doi: https://doi.org/10.1101/2020.08.27.20183012; this version posted September 1, 2020. The copyright holder for this preprint (which was not certified by peer review) is the author/funder, who has granted medRxiv a license to display the preprint in perpetuity.

It is made available under a CC-BY 4.0 International license .

\subsection{Study Selection}

Searches retrieved 4939 articles that included 965 duplicate articles, which were removed (Fig. 1). Two researchers (SGSS and DN) independently screened titles of 3974 articles and read abstracts of 442 articles, which led to exclusion of 3876 articles.. Full text of the remaining 98 articles was read independently by three researchers (SGSS, DN and VK). When recommendations differed between reviewers at the title, abstract and full text review stages, another reviewer $(\mathrm{HCvW})$ reviewed these articles and his recommendations to either include or exclude an article were final. Finally, we excluded 92 articles and included six articles in the data- extraction and narrative synthesis, and included five studies in the metaanalysis (Fig. 1).

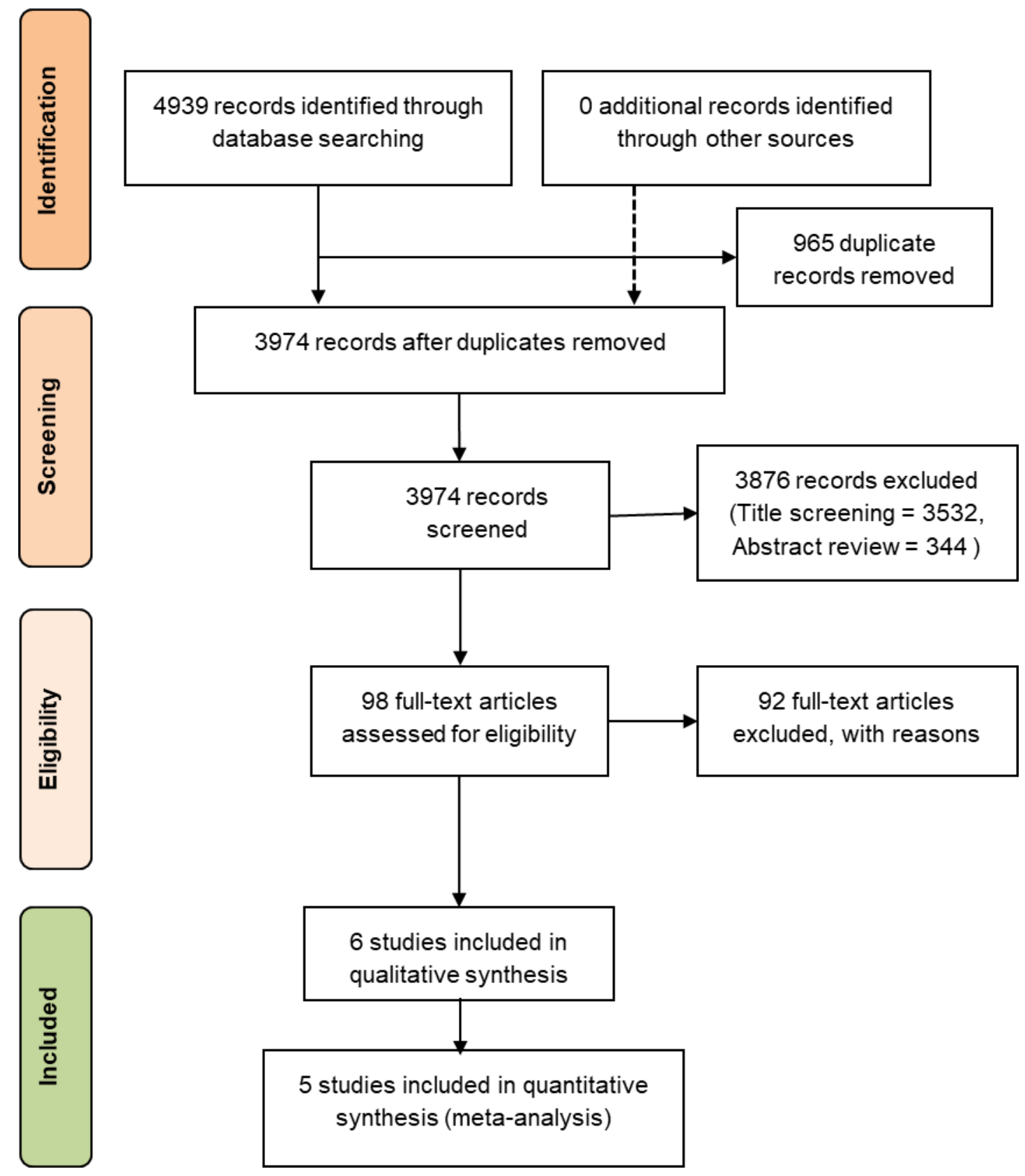

Fig. 1. PRISMA Study selection flow diagram 
medRxiv preprint doi: https://doi.org/10.1101/2020.08.27.20183012; this version posted September 1,2020 . The copyright holder for this preprint (which was not certified by peer review) is the author/funder, who has granted medRxiv a license to display the preprint in

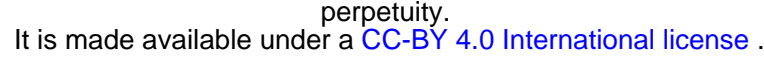

\subsection{Data collection process}

For data collection, we used a priori data extraction template (Tables 1-2), which comprised a number of columns: Author(s), year and country of study; study aim/objectives; research design; settings; participants' characteristics (age, gender and ethnicity); health/medical condition; sampling method and size; participant attrition (numbers /\%), research method(s) / data collection tool(s); intervention(s) (e.g. type/tool of digital technology), comparator(s).(e.g. alternative intervention, placebo or care as usual), intervention duration (weeks / months),. measurement stages (e.g. baseline and follow-up - weeks/months after the baseline), outcomes/results/findings (e.g. loneliness scores (including statistics e.g. mean values, standard deviations, standard errors and confidence intervals) and authors' conclusion(s) (34).

SGSS and DN independently extracted data from all included studies $(n=6)$ using the data extraction template (Tables 1-2) and resolved discrepancies in extracted data with discussion. We compared and contrasted data extraction forms; thus, we avoided bias and reduced errors in the data extraction process (41). We extracted aggregated data at the study level as much as possible with respect to the intervention, which is imperative for the reproducibility of effective interventions $(42,43)$. Following suggestions for reporting data once from studies with duplicate and multiple publications,(41) we extracted, and report, data only once (44) from a research study with multiple publications $(44,45)$.

\subsection{Data Synthesis and reporting}

We report both a narrative synthesis (narrative summary) and a statistical (quantitative) synthesis (meta-analysis) of our review study as suggested for reporting of a systematic review on the effectiveness (46). In the narrative synthesis, we include all six studies and report their characteristics including the study design, settings, sample sizes, data collection methods, participants, intervention(s), comparator(s), outcome measurements and study conclusion(s)..

In the meta-analysis, we included five studies and pooled extracted data on loneliness measured by continuous loneliness scales.

In meta-analysis, the standardised mean difference (SMD) as a summary statistic for reporting continuous data has been suggested for studies that assess the same outcome but use different scales for measuring the outcome (47). In RevMan, the SMD is the effect size known as Hedges' (adjusted) g, which is akin to Cohen's $d$ and it includes an adjustment for small sample size bias (47). In our review, the main outcome i.e. loneliness, was measured using different loneliness scales which included the UCLA loneliness scale (score range: 20$80)(48,49)$ and the de Jong Gierveld loneliness scale (score range.0-11) (50).

We calculated the standardised mean values (SMDs) from extracted data i.e. loneliness mean scores with standard deviations (SD) and sample sizes in the intervention and control groups during the follow-up measurements at three, four and six months after the intervention. We conducted meta-analysis in the Review Manager (RevMan), version 5.3.5 software (51). In the meta-analysis, we ran the random effects model as the statistical model because we hypothesised that the true effect sizes between studies would vary $(52,53)$ due to differences in the methodological and clinical characteristics between studies (54) such as 
medRxiv preprint doi: https://doi.org/10.1101/2020.08.27.20183012; this version posted September 1, 2020. The copyright holder for this preprint (which was not certified by peer review) is the author/funder, who has granted medRxiv a license to display the preprint in perpetuity.

It is made available under a CC-BY 4.0 International license .

differences in the sample sizes, participants, interventions types and duration, and the follow-up measurement times.

The Cochrane guidelines (47) suggest that different study designs should not be combined in the meta-analysis because it can increase heterogeneity and studies with repeated measurements at different follow-up periods cannot be combined without a unit-of-analysis error (47). We therefore conducted meta-analyses for similar study designs i.e. clinical trials but separated them based on the follow-up measurement periods. Thus, we undertook a separate meta-analysis for each follow-up measurement at three, four and six months after the intervention. In addition, we ran meta-analyses when there were at least two or more studies for the same outcome or the same follow-up period (55). We therefore did not run a meta-analysis for follow-up measurements at 12 months reported in two studies because they involved different study designs i.e. an RCT with intervention and control groups (40) and a pre and post intervention study with only intervention group(56) to avoid increase in the heterogeneity (47). Thus, we also avoided the overestimation of the effect of intervention in the absence of a control group (57) in the pre and post intervention study (56). In addition, we did not conduct sensitivity analyses due to the small number of studies in meta-analyses at each follow-up point (55). We had only one pre-post study and we did not run metaanalysis for this study because meta-analysis cannot be run with only one study (47).

\subsection{Assessment of research quality, bias and heterogeneity}

We assessed the quality of research (Table 3 ) by applying the GRADE (Grading of Recommendations Assessment, Development and Evaluation) approach (58).

We assessed risk of bias focusing on five domains: evaluation of sequence generation, allocation concealment, blinding (outcome assessors), incomplete data, selective outcome reporting, and assessing other biases using the Cochrane method (47). In a meta-analysis, publication bias can be assessed by graphical method using Funnel plots $(47,59)$ and statistical methods such as the Egger's test (47); however, the both methods require at least 10 studies in the meta-analysis (47). When the number of studies is less, the Egger's test has low power and it fails to differentiate chance from real asymmetry.(47). Similarly, assessing publication bias by Funnel plots with fewer studies would be of very limited usefulness because it would be difficult to spot the publication bias. We had three studies maximum in a meta-analysis (Fig. 2); hence, we could not check the publication bias by either method.

We checked heterogeneity i.e. variation in study outcomes / effect sizes between studies by the Cochran's $Q\left(\chi^{2}\right)$ Test with a significance level of $\rho<0.05(25,60)$. We used $I^{2}$ statistics for determining the magnitude of heterogeneity (i.e. the proportion of variance in the true effect sizes) between studies (25). We considered $\mathrm{I}^{2}$ of $\leq 25 \%$, between $25 \%$ and $50 \%$ and $>50 \%$ as low $(55)$, moderate $(25,61)$ and high heterogeneity between studies, respectively $(19,61)$.

\subsection{Summary measures}

We report the findings of our meta-analyses using the SMDs with 95\% confidence intervals

(Cl) as a statistical summary with the forest plots (47). 
medRxiv preprint doi: https://doi.org/10.1101/2020.08.27.20183012; this version posted September 1, 2020. The copyright holder for this preprint (which was not certified by peer review) is the author/funder, who has granted medRxiv a license to display the preprint in

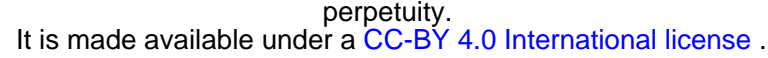

\section{RESULTS}

\subsection{Narrative synthesis}

Participants, intervention(s), comparator(s), outcome measurements and study conclusion(s) are presented in Table 1. Characteristics of studies including the study designs, settings, sample sizes, data collection methods are given in Table 2.

\subsubsection{Study selection}

The database search of PubMed, Medline, CINAHL, EMBASE, and Web of Science generated a total of 4939 papers (Fig. 1) of which six studies met the eligibility Criteria. All six studies are included in the narrative synthesis and five studies are included in the metaanalysis.

\subsubsection{Study Participants}

The total number of participants enrolled in all six included studies was 646 (mean $=108$, $S D=102$, median $=77, I Q R=32$ to 130 ). Studies varied in total sample sizes (mean 108, SD 102, range 30-300) and the sample sizes of intervention groups and control groups also varied at both the baseline and the follow-up measurements (Table 1). The attrition rate also varied between studies (range 7-35\%, mean 19\%, SD 10\%).

Participants' average age was between 73 and 78 years (SD 6-11). Total enrolled participants included 427 women (66\%) and 154 men (24\%) while no information about the gender was available for $65(10 \%)$ participants. Studies varied by gender of participants i.e. female $($ mean $=66 \%, S D=16 \%$, range $=46-81 \%$ ) and male $($ mean $=25 \%$, SD $=9 \%$, range $=$ $19-42 \%)$. Only two studies reported on participants' ethnicity, which included white (54\%) and non-whites (46\%) in the US study (40) and mostly Asian Indians (no numbers reported) in the South African study (62).

\subsubsection{Study characteristics}

The characteristics of included studies $(n=6)$ are given in Tables 2 . Of six studies included, four studies were randomised clinical trials (RCTs) $(40,44,62,63)$, one non-randomised clinical trial (NRCT) (64) and one pre- and post-test (before and after) study with intervention group only (no control group) (56) (Table 1).

\subsubsection{Study settings}

Four studies were conducted in developed countries i.e. the Netherlands (56), UK (63), US Czaja et al., 2018) and Sweden (44). Two studies were undertaken in developing countries i.e. Taiwan (64) and South Africa (62).

The settings included living in independent housing in the community (40), living in ordinary housing without any home care services (44), receiving care in their own home/supported housing in the community ("domiciliary care"), or residential care in care homes (63), residential care facilities for older people (62), nursing home (64) and elderly home care (56).

Participants were selected by random sampling in four $(60 \%)$ studies $(40,44,62,63)$ and the other two (20\%) studies used purposive (64) and convenience (56) sampling each. 
Table 1 Characteristics of included studies

\begin{tabular}{|c|c|c|c|c|c|c|c|c|c|c|c|c|c|}
\hline \multirow{2}{*}{$\begin{array}{c}\text { Authors } \\
\text { (publication } \\
\text { year) country }\end{array}$} & \multirow{2}{*}{$\begin{array}{l}\text { Quality of } \\
\text { Evidence }\end{array}$} & \multirow{2}{*}{$\begin{array}{l}\text { Research } \\
\text { Design }\end{array}$} & \multirow[t]{2}{*}{ Settings } & \multicolumn{3}{|c|}{ Participants } & \multirow{2}{*}{$\begin{array}{l}\text { Main Health } \\
\text { / Medical } \\
\text { condition(s) }\end{array}$} & \multirow{2}{*}{$\begin{array}{l}\text { Sampling } \\
\text { method }\end{array}$} & \multicolumn{3}{|c|}{ Sample size } & \multirow{2}{*}{$\begin{array}{l}\text { Participant } \\
\text { Attrition }\end{array}$} & \multirow{2}{*}{$\begin{array}{c}\begin{array}{c}\text { Research } \\
\text { Method(s)/ } \\
\text { Data } \\
\text { collection } \\
\text { tool(s) }\end{array} \\
\begin{array}{c}\text { Scale used } \\
\text { for } \\
\text { measuring } \\
\text { loneliness }\end{array}\end{array}$} \\
\hline & & & & Age (years) & Gender & Ethnicity & & & Total & $\begin{array}{l}\text { Intervention } \\
\text { group }\end{array}$ & $\begin{array}{l}\text { Control } \\
\text { group / } \\
\text { end of } \\
\text { study }\end{array}$ & & \\
\hline $\begin{array}{l}\text { Tsai et al } \\
\text { (2010) (64) } \\
\text { Taiwan }\end{array}$ & Medium & $\begin{array}{l}\text { Quasi- } \\
\text { experimental } \\
\text { study (N- } \\
\text { RCT) }\end{array}$ & $\begin{array}{l}\text { Nursing } \\
\text { home }\end{array}$ & $\begin{array}{l}\text { Baseline: } \\
\text { Experimental } \\
\text { group: } \\
\text { average age = } \\
74.2 \text { (SD } \\
10.18) ; \\
\text { Control group: } \\
\text { average age = } \\
78.48 \text { (SD } \\
6.75)\end{array}$ & $\begin{array}{l}\text { Male }=24 \\
\text { (experimental } \\
\text { group }=10, \\
\text { control group } \\
=14) ; \\
\text { Female }=33 \\
\text { (experimental } \\
\text { group }=14, \\
\text { control group } \\
=19 \text { ) }\end{array}$ & $\begin{array}{l}\text { Not reported } \\
\text { (probably all } \\
\text { Taiwanese / } \\
\text { Chinese) }\end{array}$ & $\begin{array}{l}\text { Loneliness } \\
\text { and } \\
\text { Depression }\end{array}$ & Purposive & $\begin{array}{l}57= \\
\text { Baseline, } \\
49=\text { End } \\
\text { of study }\end{array}$ & $\begin{array}{l}24= \\
\text { Baseline, } \\
21=\text { Follow- } \\
\text { up }\end{array}$ & $\begin{array}{l}33= \\
\text { Baseline } \\
, .28= \\
\text { Follow- } \\
\text { up }\end{array}$ & $\begin{array}{l}\text { Total = 8 (5 } \\
\text { from control } \\
\text { group and } 3 \\
\text { from } \\
\text { experimental } \\
\text { group), } \\
\text { attrition rate } \\
=14 \%\end{array}$ & $\begin{array}{l}\text { UCLA } \\
\text { Loneliness } \\
\text { Scale } \\
\text { (Russel et } \\
\text { al, 1980) }\end{array}$ \\
\hline $\begin{array}{l}\text { van der Heide } \\
\text { et al (2012) } \\
\text { (56) The } \\
\text { Netherlands }\end{array}$ & Low & $\begin{array}{l}\text { Before and } \\
\text { after study } \\
\text { (with } \\
\text { intervention } \\
\text { group only, } \\
\text { no control } \\
\text { group) }\end{array}$ & $\begin{array}{l}\text { Elderly } \\
\text { home care }\end{array}$ & $\begin{array}{l}\text { Baseline: } \\
\text { average } 73.2 \\
\text { (SD 11.8), } \\
\text { range 32-90; } \\
\text { End of study } \\
\text { stage: } \\
\text { Average } 73.1 \\
\text { (SD 11.2), } \\
\text { range 38-90 }\end{array}$ & $\begin{array}{l}\text { Baseline: Male = } \\
26(30.2 \%), \\
\text { Female }=60 \\
(69.8 \%), \text { Missing } \\
\text { values } n=44 ; \\
\text { End of study: } \\
\text { Male }=25 \\
(29.4 \%), \text { Female } \\
=60(70.6 \%), \\
\text { missing } \\
\text { values. }=0\end{array}$ & Not reported & $\begin{array}{l}\text { Feeling of } \\
\text { loneliness } \\
\text { and safety }\end{array}$ & $\begin{array}{l}\text { Convenienc } \\
\text { e }\end{array}$ & 130 & 130 & $\begin{array}{l}\text { intervent } \\
\text { ion } \\
\text { group at } \\
\text { the end } \\
\text { of study } \\
=85 \text {. } \\
\text { No } \\
\text { control } \\
\text { group }\end{array}$ & $\begin{array}{l}\text { Total }=45, \\
\text { attrition rate } \\
=34.6 \%\end{array}$ & $\begin{array}{l}\text { De Jong- } \\
\text { Gierveld } \\
\text { loneliness } \\
\text { scale (Score } \\
\text { range: } 0-11 \text { ) }\end{array}$ \\
\hline $\begin{array}{l}\text { Larsson et al } \\
\text { (2016) (44) } \\
\text { Sweden }\end{array}$ & High & $\begin{array}{l}\text { Randomise, } \\
\text { crossover } \\
\text { study }\end{array}$ & $\begin{array}{l}\text { Living in } \\
\text { ordinary } \\
\text { housing } \\
\text { without any } \\
\text { home care } \\
\text { services }\end{array}$ & $\begin{array}{l}\text { Total sample: } \\
\text { range: 61-89 } \\
\text { (SD 71.2); } \\
\text { Group 1 } \\
\text { (intervention / } \\
\text { control group): } \\
\text { range 66-89 } \\
\text { (SD 73.6); } \\
\text { Group 2 } \\
\text { (Control/interv } \\
\text { ention group): } \\
\text { range 61-76 } \\
\text { (SD 69.0) }\end{array}$ & $\begin{array}{l}\text { Total: male =6, } \\
\text { female }=24,(3 \\
\text { males and } 12 \\
\text { females each in } \\
\text { Group } 1 \\
\text { (intervention / } \\
\text { control group) } \\
\text { and Group } 2 \\
\text { (Control / } \\
\text { intervention } \\
\text { group) }\end{array}$ & $\begin{array}{l}\text { Not reported } \\
\text { (probably all } \\
\text { Swedes) }\end{array}$ & Loneliness & $\begin{array}{l}\text { Randomised } \\
\text { (after } \\
\text { recruitment) }\end{array}$ & 30 & $\begin{array}{l}15= \\
\text { Baseline, } \\
14=\text { Follow- } \\
\text { up }\end{array}$ & $\begin{array}{l}15= \\
\text { Baseline } \\
14= \\
\text { Follow- } \\
\text { up }\end{array}$ & $\begin{array}{l}\text { Total = 2(1 } \\
\text { participant } \\
\text { each from } \\
\text { intervention } \\
\text { and control } \\
\text { groups), } \\
\text { attrition rate } \\
=6.7 \%\end{array}$ & $\begin{array}{l}\text { UCLA } \\
\text { loneliness } \\
\text { scale } \\
\text { (Russell, } \\
\text { 1996) - the } \\
\text { Swedish } \\
\text { version } \\
\text { (Engelberg } \\
\text { and Sjoberg } \\
(2005) \\
\text { (20 items, } \\
\text { score range } \\
20-80)\end{array}$ \\
\hline
\end{tabular}




\begin{tabular}{|c|c|c|c|c|c|c|c|c|c|c|c|c|c|}
\hline $\begin{array}{l}\text { Czaja et al } \\
\text { (2018) (40) } \\
\text { United States }\end{array}$ & High & $\begin{array}{l}\text { Multisite } \\
\text { randomised } \\
\text { controlled } \\
\text { trial }\end{array}$ & $\begin{array}{l}\text { Living in } \\
\text { independent } \\
\text { housing in } \\
\text { the } \\
\text { community }\end{array}$ & $\begin{array}{l}\text { Baseline: total } \\
\text { sample mean } \\
=76.15 \text { (SD } \\
7.4), \text { range: } \\
65-98 ; \\
\text { Intervention } \\
\text { (PRISM) } \\
\text { group: mean = } \\
76.9 \text { (SD 7.3); } \\
\text { Control } \\
\text { (Binder) } \\
\text { group: mean = } \\
75.3 \text { (SD 7.4) }\end{array}$ & $\begin{array}{l}\text { Baseline: } \\
\text { Female = 78\% } \\
\text { (number not } \\
\text { reported), Male } \\
=22 \% \text { (number } \\
\text { not reported); } \\
\text { PRISM/Intervent } \\
\text { ion group: } \\
\text { Female 79.3\% } \\
\text { ( } n=119) ; \text { Binder } \\
\text { (control) group: } \\
\text { Female 76.7\% } \\
\text { ( } n=115)\end{array}$ & $\begin{array}{l}\text { Baseline: } \\
\text { White } 54 \% \\
\text { and Non- } \\
\text { white (46\%); } \\
\text { PRISM/Inter } \\
\text { vention } \\
\text { group: Non- } \\
\text { white/Hispan } \\
\text { ic } n=12,8 \% \text {; } \\
\text { Binder } \\
\text { group: Non- } \\
\text { white/Hispan } \\
\text { ic } n=15 \text {, } \\
10.0 \%\end{array}$ & $\begin{array}{l}\text { Social } \\
\text { isolation, } \\
\text { Social } \\
\text { support, } \\
\text { Loneliness, } \\
\text { and } \\
\text { Wellbeing }\end{array}$ & Randomised & $\begin{array}{l}300(150 \\
\text { in each } \\
\text { interventio } \\
\mathrm{n} \text { arm } \\
\text { (PRISM } \\
\text { group) } \\
\text { and } \\
\text { control } \\
\text { arm } \\
\text { (Binder } \\
\text { group) }\end{array}$ & $\begin{array}{l}150= \\
\text { Baseline, } \\
134= \\
\text { Follow-up }\end{array}$ & $\begin{array}{l}150= \\
\text { Baseline } \\
, 118= \\
\text { Follow- } \\
\text { up }\end{array}$ & $\begin{array}{l}\text { Total }=56, \\
\text { ( } 45 \text { at } 6 \\
\text { months and } \\
11 \text { at } 12 \\
\text { month } \\
\text { follow-up), } \\
\text { Attrition rate } \\
=18.7 \%\end{array}$ & $\begin{array}{l}\text { UCLA } \\
\text { Loneliness } \\
\text { Scale } \\
\text { (Russell, } \\
\text { 1996) (score } \\
\text { range 20-80) }\end{array}$ \\
\hline $\begin{array}{l}\text { Morton et al } \\
\text { (2018) (63) } \\
\text { United } \\
\text { Kingdom }\end{array}$ & High & $\begin{array}{l}2 \text { (condition: } \\
\text { training, } \\
\text { control) } \times 2 \\
\text { (population: } \\
\text { domiciliary, } \\
\text { residential) } \\
\times 2 \text { (time: } \\
\text { baseline, } \\
\text { follow-up) } \\
\text { design }\end{array}$ & $\begin{array}{l}\text { Receiving } \\
\text { care in own } \\
\text { home / } \\
\text { supported } \\
\text { housing in } \\
\text { the } \\
\text { community } \\
\text { ("domiciliary } \\
\text { care"), or } \\
\text { residential } \\
\text { care in care } \\
\text { homes }\end{array}$ & $\begin{array}{l}\text { Female: mean } \\
=80.71 \text { (SD } \\
=8.77) \\
\text { Male: data not } \\
\text { reported }\end{array}$ & $\begin{array}{l}\text { Follow-up: Total } \\
=76 ; \text { Female }= \\
50, \text { Male }=26\end{array}$ & Not reported & $\begin{array}{l}\text { Wellbeing } \\
\text { and social } \\
\text { support }\end{array}$ & Randomised & $\begin{array}{l}97= \\
\text { Baseline, } \\
76= \\
\text { Follow-up }\end{array}$ & $\begin{array}{l}53= \\
\text { Baseline, } 44 \\
=\text { Follow-up }\end{array}$ & $\begin{array}{l}44= \\
\text { Baseline } \\
, 32= \\
\text { Follow- } \\
\text { up }\end{array}$ & $\begin{array}{l}\text { Total = } 21(9 \\
= \\
\text { experimental } \\
\text { group, } 12= \\
\text { control } \\
\text { group) } \\
\text { attrition rate } \\
=21.6 \%\end{array}$ & $\begin{array}{l}\text { UCLA } \\
\text { Loneliness } \\
\text { Scale } \\
\text { (Russell, } \\
\text { 1996) (score } \\
\text { range 20- } \\
80 \text { ) }\end{array}$ \\
\hline $\begin{array}{l}\text { Jarvis et al } \\
\text { (2019) (62) } \\
\text { South Africa }\end{array}$ & High & $\begin{array}{l}\text { Randomised } \\
\text { control study }\end{array}$ & $\begin{array}{l}\text { Inner-city } \\
\text { residential } \\
\text { NGO care } \\
\text { facilities for } \\
\text { resource- } \\
\text { restricted } \\
\text { older people } \\
\text { ( } \geq 60 \text { years) }\end{array}$ & $\begin{array}{l}\text { Mean }=74.93 \\
(S D 6.41) \\
\text { range = 61-87 }\end{array}$ & $\begin{array}{l}\text { Baseline: Male = } \\
6(18.8 \%) ; \\
\text { Female =26 } \\
(81.2 \%)\end{array}$ & $\begin{array}{l}\text { Mostly Asian } \\
\text { / Indian, } \\
\text { numbers not } \\
\text { reported }\end{array}$ & $\begin{array}{l}\text { maladaptive } \\
\text { cognitions } \\
\text { and } \\
\text { loneliness }\end{array}$ & Randomised & $\begin{array}{l}2= \\
\text { Baseline } \\
\text { (interventi } \\
\text { on group } \\
=15, \\
\text { control } \\
\text { group } \\
=17 \text { ), } \\
\text { Final = } 29 \\
\text { (interventi } \\
\text { on group } \\
==13, \\
\text { control } \\
\text { group } \\
=16 \text { ) }\end{array}$ & $\begin{array}{l}15= \\
\text { Baseline, } 13 \\
=\text { Follow-up }\end{array}$ & $\begin{array}{l}17= \\
\text { Baseline } \\
, 16 \\
=\text { Follow- } \\
\text { up }\end{array}$ & $\begin{array}{l}\text { Total = } 3 \text { (2 } \\
= \\
\text { intervention } \\
\text { group, } 1= \\
\text { control } \\
\text { group), } \\
\text { Attrition rate } \\
=15.6 \%\end{array}$ & $\begin{array}{l}\text { De Jong- } \\
\text { Gierveld } \\
\text { loneliness } \\
\text { scale (Score } \\
\text { range: } 0-11 \text { ) }\end{array}$ \\
\hline
\end{tabular}


medRxiv preprint doi: https://doi.org/10.1101/2020.08.27.20183012; this version posted September 1, 2020. The copyright holder for this preprint (which was not certified by peer review) is the author/funder, who has granted medRxiv a license to display the preprint in perpetuity.
It is made available under a CC-BY 4.0 International license.

\subsubsection{Digital technology interventions}

Digital technology interventions included social internet-based activities (SIBAs) such as Skype and Facebook (44), videoconferencing (64), customized computer platform with simplified touch-screen interface (63), personal reminder information and social management system (40), WhatsApp group (62) and video/voice network (56).

\subsubsection{Duration of intervention and measurement of main outcome measure}

The duration of intervention was three months in four studies $(44,62-64)$ and 12 months in two studies $(40,56)$. The main outcome measure i.e. loneliness was measured at the baseline and multiple follow-up times, which included at three months in three studies (44, $62,64)$, four months in two studies $(62,63)$, six months in two studies $(40,44)$ and 12 months in two studies $(40,56)$. Loneliness measurement tools were the UCLA loneliness scale $(48,49)$ used in four studies $(40,44,63,64)$ and the De Jong-Gierveld loneliness questionnaire $(50,65)$ used in two studies $(56,62)$. Table 2 presents loneliness scores measured in the intervention and control groups, if any, at the baseline and follow-ups. Narrative synthesis showed that there was reduction in loneliness in the intervention groups at the follow-ups compared to at the baseline (Table 2). A statistical summary of the loneliness measurements in the intervention and control groups at the follow-ups is reported in the meta-analysis section. 
Table 2 Interventions and outcome measurements in included studies

\begin{tabular}{|c|c|c|c|c|c|c|c|c|c|c|c|}
\hline \multirow[t]{2}{*}{ Study } & \multirow[t]{2}{*}{ Intervention(s) } & \multirow[t]{2}{*}{ Comparator(s) } & \multirow[t]{2}{*}{$\begin{array}{l}\text { Intervention } \\
\text { duration }\end{array}$} & \multirow[t]{2}{*}{$\begin{array}{l}\text { Follow-up } \\
\text { duration }\end{array}$} & \multicolumn{5}{|c|}{$\begin{array}{l}\text { Outcomes: Loneliness scores by measurement stages } \\
\qquad \text { Mean Scores (SD) }\end{array}$} & \multirow[t]{2}{*}{ Results / Findings } & \multirow[t]{2}{*}{$\begin{array}{l}\text { Authors' } \\
\text { Conclusion }\end{array}$} \\
\hline & & & & & Baseline & 3 months & 4 months & 6 months & 12 months & & \\
\hline $\begin{array}{l}\text { Tsai et al } \\
(2010)(64)\end{array}$ & $\begin{array}{l}\text { Videoconference } \\
\text { interaction (using } \\
\text { either MSN or } \\
\text { Skype) }\end{array}$ & Regular care & 3 months & 3 months & $\begin{array}{l}\text { Intervention } \\
\text { group = } \\
50.58(S D \\
11.16) \\
\text { Control } \\
\text { group = } \\
46.55 \text { (SD } \\
9.07)\end{array}$ & $\begin{array}{l}\text { Intervention } \\
\text { group = } \\
47.33(\mathrm{SD}= \\
13.50) ; \\
\text { Control } \\
\text { group = } \\
46.68(\mathrm{SD}= \\
9.08)\end{array}$ & $\begin{array}{l}\text { Not } \\
\text { measured }\end{array}$ & $\begin{array}{l}\text { Not } \\
\text { measured }\end{array}$ & $\begin{array}{l}\text { Not } \\
\text { measured }\end{array}$ & $\begin{array}{l}\text { Loneliness: Intervention group mean } \\
(\text { SD) = baseline } 50.58 \text { ( } 11.16) \text {, one } \\
\text { week } 49.75 \text { (11.79) and } 3 \text { months } \\
47.33 \text { (13.50); Control group mean } \\
(\mathrm{SD})=\text { baseline } 46.55 \text { (9.07), one } \\
\text { week } 47.06 \text { (8.75) and } 3 \text { months } \\
46.68 \text { (9.08); Differences between } \\
\text { groups were compared at three } \\
\text { points (baseline, one week, and } \\
\text { three months) using multiple linear } \\
\text { regression of the generalized } \\
\text { estimating equations. Unadjusted / } \\
\text { fixed effect of effectiveness of } \\
\text { videoconferencing intervention } \\
\text { videoconference vs. control: at one } \\
\text { week Beta }(\beta)=-1.21 . \text { SE } 0.50, \chi^{2} \\
=5.95, p=0.02 \text { and at } 3 \text { months } \\
\text { Beta }(\beta)=-2.84, S E 1.28, \chi^{2}=4.89, \\
p=0.03 \text {. }\end{array}$ & $\begin{array}{l}\text { Videoconferen } \\
\text { ce program } \\
\text { alleviate } \\
\text { depressive } \\
\text { symptoms and } \\
\text { loneliness in } \\
\text { elderly } \\
\text { residents in } \\
\text { nursing homes }\end{array}$ \\
\hline $\begin{array}{l}\text { van der } \\
\text { Heide et al } \\
(2012)(56)\end{array}$ & $\begin{array}{l}\text { CareTV } \\
\text { intervention } \\
\text { including Caret } \\
\text { duplex } \\
\text { video/voice } \\
\text { network }\end{array}$ & $\begin{array}{l}\text { No control } \\
\text { group and no } \\
\text { comparator }\end{array}$ & 12 months & $\begin{array}{l}12 \\
\text { months }\end{array}$ & $\begin{array}{l}\text { Interventio } \\
\text { n group = } \\
5.97 \text { (SD } \\
2.77) ; \\
\text { No control } \\
\text { group }\end{array}$ & $\begin{array}{l}\text { Not } \\
\text { measured }\end{array}$ & $\begin{array}{l}\text { Not } \\
\text { measured }\end{array}$ & $\begin{array}{l}\text { Not } \\
\text { measured }\end{array}$ & $\begin{array}{l}\text { Interventio } \\
\mathrm{n} \text { group = } \\
4.02 \text { (SD } \\
3.91) ; \text { No } \\
\text { control } \\
\text { group }\end{array}$ & $\begin{array}{l}\text { Group level total loneliness: } \\
\text { Inclusion stage: mean } 5.97 \text { (SD } \\
2.77) \text { (scale 0-11), End of study: } \\
\text { mean } 4.02 \text { (SD 3.91), p = } 0.001 \text {. } \\
\text { Individual level total Loneliness: } \\
\text { total loneliness decreased in } 54 \\
\text { out of } 85 \text { participants (equally } \\
\text { lonely } 11 \text {, more lonely } 20 \text { and } \\
\text { less lonely } 54 \text { individual } \\
\text { participants) }\end{array}$ & $\begin{array}{l}\text { CareTV } \\
\text { intervention } \\
\text { decreased } \\
\text { the feeling of } \\
\text { loneliness in } \\
\text { the } \\
\text { participants; } \\
\text { however, } \\
\text { participants } \\
\text { were feeling } \\
\text { moderate } \\
\text { loneliness at } \\
\text { the end of } \\
\text { the study }\end{array}$ \\
\hline
\end{tabular}




\begin{tabular}{|c|c|c|c|c|c|c|c|c|c|c|c|}
\hline $\begin{array}{l}\text { Larsson et } \\
\text { al } \\
(2016)(44)\end{array}$ & $\begin{array}{l}\text { Social internet- } \\
\text { based activities } \\
\text { (SIBAs)such as } \\
\text { Skype and } \\
\text { Facebook }\end{array}$ & $\begin{array}{l}\text { No comparator } \\
\text { intervention } \\
\text { reported }\end{array}$ & 3 months & $\begin{array}{l}34 \text { weeks } \\
\text { (3 } \\
\text { months } \\
\text { exposure } \\
\text { to each } \\
\text { group) }\end{array}$ & $\begin{array}{l}\text { Group } 1 \\
(\mathrm{I} / \mathrm{C} \\
\text { group) }= \\
45.53 \\
(\mathrm{SD}=7.41) \\
\text { Group } 2 \\
\text { (C/I group) } \\
=43.93 \\
\text { (SD 8.61) }\end{array}$ & $\begin{array}{l}\text { Group } 1 \\
(\mathrm{I} / \mathrm{C} \\
\text { group) }= \\
42.43 \\
(\mathrm{SD}=7.44) \\
\text { Group } 2 \\
\text { (C/I group) } \\
=41.93 \\
\text { (SD 8.82), }\end{array}$ & $\begin{array}{l}\text { Not } \\
\text { measured }\end{array}$ & $\begin{array}{l}\text { months } \\
\text { after cross } \\
\text { over: } \\
\text { Group } 1 \\
\text { (I/C group, } \\
\text { No } \\
\text { interventio } \\
\mathrm{n})=42.0 \\
(\mathrm{SD}=7.34) \text {; } \\
\text { Group } 2 \\
\text { (C/I group, } \\
\text { Interventio } \\
\mathrm{n} \\
\text { introduced) } \\
=39.50 \\
\text { (SD } \\
10.42) \text {. }\end{array}$ & $\begin{array}{l}\text { Not } \\
\text { measured }\end{array}$ & $\begin{array}{l}\text { \% change between T2 and T1: } \\
\text { Group } 1=\text { mean score : }-0.07 \% \\
\text { (SD 0.07), p. } 0.003 ; \text { Group } 2= \\
\text { mean score : }-0.05 \% \text { (SD } 0.09 \text { ), } \\
\text { p. } 0.049 ; \\
\% \text { change between T3 and T1: } \\
\text { Group } 1=\text { mean score : }-0.08 \% \\
\text { (SD 0.08); Group } 2=\text { mean score } \\
:-0.09 \% \text { (SD 0.13); } \\
\text { Comparison of pre and post- } \\
\text { intervention scores group } 1=\text { p. } \\
0.003 \text { and group } 2=\text { p. } 0.049\end{array}$ & $\begin{array}{l}\text { SIBA } \\
\text { interventions } \\
\text { have } \\
\text { potential to } \\
\text { reduce } \\
\text { experiences } \\
\text { of loneliness } \\
\text { in socially } \\
\text { vulnerable } \\
\text { older adults. }\end{array}$ \\
\hline $\begin{array}{l}\text { Czaja et al } \\
(2018)(40)\end{array}$ & $\begin{array}{l}\text { Personal } \\
\text { Reminder } \\
\text { Information and } \\
\text { Social } \\
\text { Management } \\
\text { (PRISM) } \\
\text { system }\end{array}$ & $\begin{array}{l}\text { A notebook } \\
\text { with printed } \\
\text { content similar } \\
\text { to that within } \\
\text { PRISM group } \\
\text { (intervention } \\
\text { group) - } \\
\text { include a } \\
\text { Lenovo "Mini } \\
\text { Desktop" PC } \\
\text { with a } \\
\text { keyboard, } \\
\text { mouse (or } \\
\text { trackball for } \\
\text { those who } \\
\text { were unable to } \\
\text { control a } \\
\text { mouse), a 19" } \\
\text { LCD monitor, } \\
\text { the PRISM } \\
\text { software } \\
\text { application, } \\
\text { and a printer } \\
\text { and internet } \\
\text { access. }\end{array}$ & 12 months & $\begin{array}{l}12 \\
\text { months }\end{array}$ & $\begin{array}{l}\text { Interventio } \\
\text { n (PRISM) } \\
\text { group = } \\
39.8 \text { (SD } \\
9.7) ; \\
\text { Control } \\
\text { (Binder) } \\
\text { group = } \\
40.2 \text { (SD } \\
10.3)\end{array}$ & $\begin{array}{l}\text { Not } \\
\text { measured }\end{array}$ & $\begin{array}{l}\text { Not } \\
\text { measured }\end{array}$ & $\begin{array}{l}\text { Interventio } \\
\text { n (PRISM) } \\
\text { group }= \\
37.8 \text { (SD } \\
9.54) ; \\
\text { Control } \\
\text { (Binder) } \\
\text { group = 40 } \\
\text { (SD 10.62) }\end{array}$ & $\begin{array}{l}\text { Interventio } \\
\text { n (PRISM) } \\
\text { group = } \\
36.9 \text { (SD } \\
9.16) ; \\
\text { Control } \\
\text { (Binder) } \\
\text { group = } \\
38.43 \text { (SD } \\
9.37)\end{array}$ & $\begin{array}{l}\text { Baseline: Loneliness = PRISM } \\
\text { group mean score } 39.8 \text { (SD 9.7) } \\
\text { and Binder group mean score } \\
40.2 \text { (SD 10.3), follow-up at } 6 \\
\text { months PRISM group about } \\
37.8 \text { and Binder group } 39.6 ; \\
\text { Follow-up at } 12 \text { months PRISM } \\
\text { group } 36.9 \text { and Binder group } \\
38.3 \text {. }\end{array}$ & $\begin{array}{l}\text { Technology } \\
\text { applications } \\
\text { such as } \\
\text { PRISM may } \\
\text { enhance } \\
\text { social } \\
\text { connectivity } \\
\text { and reduce } \\
\text { loneliness } \\
\text { among older } \\
\text { adults. }\end{array}$ \\
\hline
\end{tabular}




\begin{tabular}{|c|c|c|c|c|c|c|c|c|c|c|c|}
\hline $\begin{array}{l}\text { Morton et } \\
\text { al } \\
(2018)(63)\end{array}$ & $\begin{array}{l}\text { "EasyPC" - a } \\
\text { customized } \\
\text { computer } \\
\text { platform with a } \\
\text { simplified } \\
\text { touch-screen } \\
\text { interface } \\
\text { (Intervention } \\
\text { group) }\end{array}$ & $\begin{array}{l}\text { Care as usual } \\
\text { (plus regular } \\
\text { carer visits) }\end{array}$ & 3 months & 3 months & $\begin{array}{l}\text { Interventio } \\
\mathrm{n} \\
\text { (Training) } \\
\text { group (total } \\
\text { of } \\
\text { residential } \\
\text { and } \\
\text { domiciliary } \\
\text { groups): } \\
=1.95 \text { (SD: } \\
0.73 \text { ); } \\
\text { Control } \\
\text { group (total } \\
\text { of } \\
\text { residential } \\
\text { and } \\
\text { domiciliary } \\
\text { groups): } \\
=2.08 \text { (SD } \\
0.80 \text { ) }\end{array}$ & $\begin{array}{l}\text { Not } \\
\text { measured }\end{array}$ & $\begin{array}{l}\text { Interventio } \\
\mathrm{n} \\
\text { (Training) } \\
\text { group (total } \\
\text { of } \\
\text { residential } \\
\text { and } \\
\text { domiciliary } \\
\text { groups): } \\
1.86 \text { (SD } \\
0.66 \text { ); } \\
\text { Control } \\
\text { group (total } \\
\text { of } \\
\text { residential } \\
\text { and } \\
\text { domiciliary } \\
\text { groups): } \\
2.12 \text { (SD } \\
0.62 \text { ) }\end{array}$ & $\begin{array}{l}\text { Not } \\
\text { measured }\end{array}$ & $\begin{array}{l}\text { Not } \\
\text { measured }\end{array}$ & 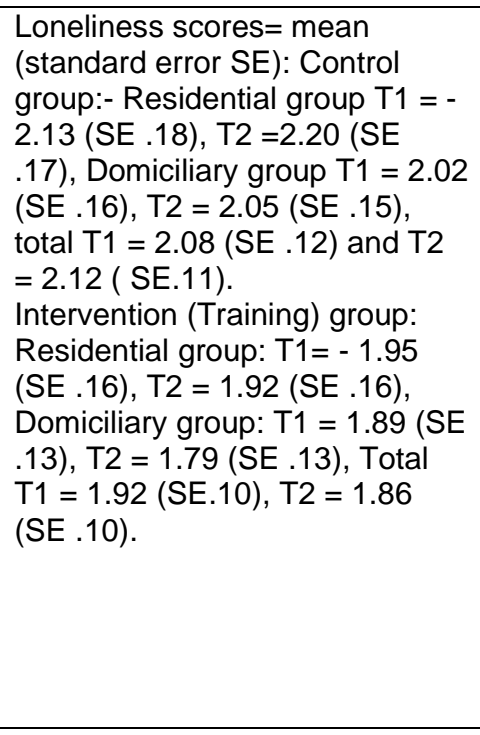 & $\begin{array}{l}\text { Internet } \\
\text { access and } \\
\text { training can } \\
\text { support the } \\
\text { self and } \\
\text { social } \\
\text { connectedne } \\
\text { ss of } \\
\text { vulnerable } \\
\text { older adults } \\
\text { and } \\
\text { contribute } \\
\text { positively to } \\
\text { well-being. }\end{array}$ \\
\hline $\begin{array}{l}\text { Jarvis et al } \\
(2019)(62)\end{array}$ & $\begin{array}{l}\text { Living In } \\
\text { Network } \\
\text { Connected } \\
\text { Communities } \\
\text { (mLINCC) } \\
\text { WhatsApp } \\
\text { group for low- } \\
\text { intensity } \\
\text { Cognitive } \\
\text { Behavior } \\
\text { Therapy (LI- } \\
\text { CBT) } \\
\text { intervention }\end{array}$ & $\begin{array}{l}\text { Usual care, a } \\
\text { separate } \\
\text { WhatsApp } \\
\text { group } \\
\text { (mLINCC 2) }\end{array}$ & 3 months & $\begin{array}{l}3-4 \\
\text { months }\end{array}$ & $\begin{array}{l}\text { Not } \\
\text { measured }\end{array}$ & $\begin{array}{l}\text { Interventio } \\
\text { n group = } \\
2.31(S D= \\
1.33) ; \\
\text { Control } \\
\text { group = } \\
2.47(\mathrm{SD}= \\
2.1)\end{array}$ & $\begin{array}{l}\text { Interventio } \\
\mathrm{n} \text { group = } \\
1.38(\mathrm{SD}= \\
1.33) ; \\
\text { Control } \\
\text { group = } \\
4.0(\mathrm{SD}= \\
1.32)\end{array}$ & $\begin{array}{l}\text { Not } \\
\text { measured }\end{array}$ & $\begin{array}{l}\text { Not } \\
\text { measured }\end{array}$ & $\begin{array}{l}\text { Ioneliness levels: Total }=\mathrm{T} 0 \\
\mathrm{~T} 1-\mathrm{T} 2, \chi^{2}=14.62, \mathrm{p}=0.001 \text { ) }\end{array}$ & $\begin{array}{l}\text { LI-CBT } \\
\text { mHealth } \\
\text { supported by } \\
\text { the social } \\
\text { networking } \\
\text { platform of } \\
\text { WhatsApp } \\
\text { (mLINCC) } \\
\text { showed } \\
\text { significant } \\
\text { improvement } \\
\text { s in } \\
\text { loneliness } \\
\text { and } \\
\text { maladaptive } \\
\text { cognitions. }\end{array}$ \\
\hline
\end{tabular}


medRxiv preprint doi: https://doi.org/10.1101/2020.08.27.20183012; this version posted September 1, 2020. The copyright holder for this preprint (which was not certified by peer review) is the author/funder, who has granted medRxiv a license to display the preprint in It is made available under a CC-BY 4.0 International license .

\subsection{Meta-analysis}

We ran three meta-analyses one each for follow-up measurements at three, four and six months involving three, two and two studies respectively.

\subsubsection{Meta-analysis for follow up measurements at $\mathbf{3}$ months}

Three studies $(44,62,64)$ involving 106 participants with follow-up measurement at three months were entered into a meta-analysis, which showed a very small reduction in loneliness in favour of the control $(S M D=0.02,95 \% \mathrm{Cl}-0.36,0.40)$ but it was not statistically significant $(Z=0.10, P=0.92)$. The heterogeneity between studies was not statistically significant $\left(\mathrm{T}^{2}=0.00, \chi^{2}=0.10, P=0.95, \mathrm{I}^{2}=0 \%\right)($ Fig. 2 - panel $\mathrm{A})$.

\subsection{Meta-analysis for follow up at 4 months}

Two studies $(62,63)$ involving 105 participants with four month follow-up were entered into a meta-analysis, which revealed a large reduction in loneliness in favour of the intervention $(S M D=-1.11,95 \% \mathrm{Cl}-2.60,0.38)$ but was not statistically significant $(Z=1.46 ; P=0.14)$. There was statistically significant high heterogeneity between studies $\left(\mathrm{T}^{2}=1.03, \chi^{2}=8.84, P=0.003\right.$, $\mathrm{I}^{2}=88 \%$ ) (Fig. 2 - panel B).

\subsection{Meta-analysis for follow up measurements at 6 months}

A meta-analysis involving 2 studies $(40,44)$ with 208 participants with six month follow-up showed a very small reduction in loneliness in favour of the intervention (SMD $=-0.11$, $95 \% \mathrm{Cl}-0.54,0.32)$ but it was not statistically significant $(Z=0.51, P=0.61)$. There was moderate heterogeneity between studies but not statistically significant $\left(\mathrm{T}^{2}=0.05, \chi^{2}=1.58\right.$, $\mathrm{P}=0.21, \mathrm{I}^{2}=37 \%$ ) (Fig. 2- panel C). 


\section{A. Follow-up: 3 months after}

\begin{tabular}{|c|c|c|c|c|c|c|c|c|c|}
\hline \multirow[b]{2}{*}{ Study or Subgroup } & \multicolumn{3}{|c|}{ Intervention } & \multicolumn{3}{|c|}{ Control } & \multicolumn{2}{|r|}{ Std. Mean Difference } & \multirow{2}{*}{$\begin{array}{l}\text { Std. Mean Difference } \\
\text { IV, Random, } 95 \% \mathrm{Cl}\end{array}$} \\
\hline & Mean & SD & Total & Mean & SD & Total & Weight & IV, Random, $95 \% \mathrm{Cl}$ & \\
\hline Jarvis et al 2019 & 2.31 & 1.49 & 13 & 2.47 & 2.1 & 16 & $27.4 \%$ & $-0.08[-0.82,0.65]$ & \\
\hline Larsson et al 2016 & 42.43 & 7.44 & 14 & 41.93 & 8.82 & 14 & $26.7 \%$ & $0.06[-0.68,0.80]$ & \\
\hline Tsai et al 2010 & 47.33 & 13.5 & 21 & 46.68 & 9.08 & 28 & $45.9 \%$ & $0.06[-0.51,0.62]$ & \\
\hline Total $(95 \% \mathrm{Cl})$ & & & 48 & & & 58 & $100.0 \%$ & $0.02[-0.36,0.40]$ & \\
\hline $\begin{array}{l}\text { Heterogeneity: Tau² } \\
\text { Test for overall effect }\end{array}$ & $\begin{array}{l}0.00 ; C l \\
Z=0.10\end{array}$ & $\begin{array}{l}h i^{2}=0 \\
(P=0\end{array}$ & $\begin{array}{l}10, \mathrm{df}= \\
9.92)\end{array}$ & $=2(\mathrm{P}=$ & $0.95) ;$ & $\left.\right|^{2}=0 \%$ & & & $\begin{array}{ccccc} & 1 & 1 & 1 & 2 \\
\text { Favours [Intervention] } & \text { Favours [Control] }\end{array}$ \\
\hline
\end{tabular}

\section{B. Follow-up: 4 months after}

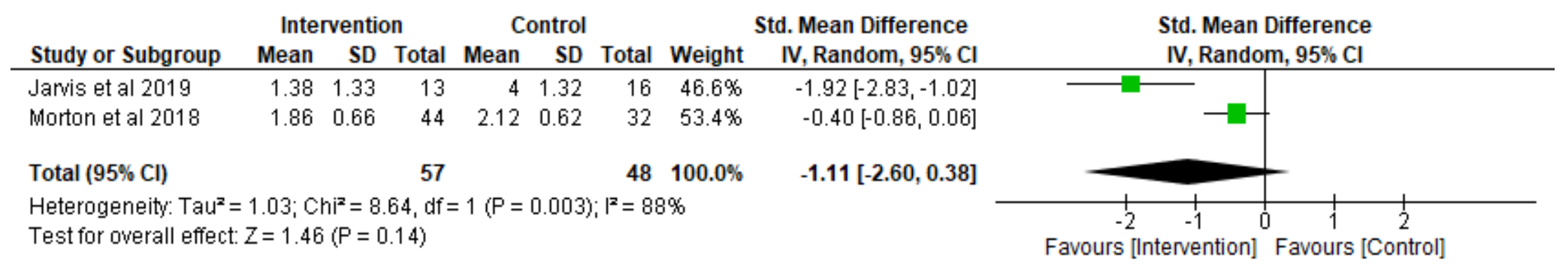

\section{Follow-up: 6 months after}

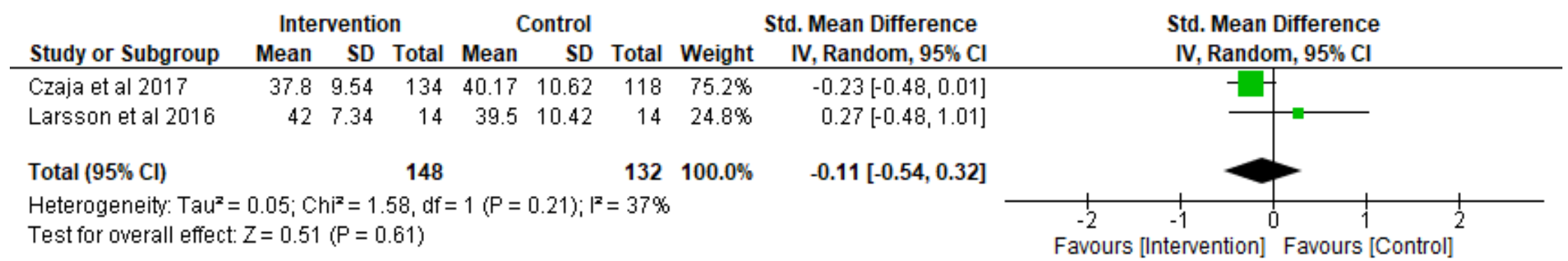

Fig. 2 Forest plots Comparison: Digital technology Intervention vs Control, Outcome: Loneliness 
medRxiv preprint doi: https://doi.org/10.1101/2020.08.27.20183012; this version posted September 1, 2020. The copyright holder for this preprint (which was not certified by peer review) is the author/funder, who has granted medRxiv a license to display the preprint in perpetuity.

It is made available under a CC-BY 4.0 International license.

\subsection{Risk of Bias}

Risk of bias assessment is presented in Figs. 3-4. A high risk of bias was noted more in the attrition bias and other bias; unclear risk of bias was detected in blinding of outcome assessment, allocation concealment and blinding of participants and personnel while a low risk of bias was observed especially in the random sequence generation and selective reporting (Fig. 3). In addition, most studies reported only within-group changes and not between-group comparisons of change, which may suggest a weak quality of the reporting of results and the analysis in these studies (Table 2).

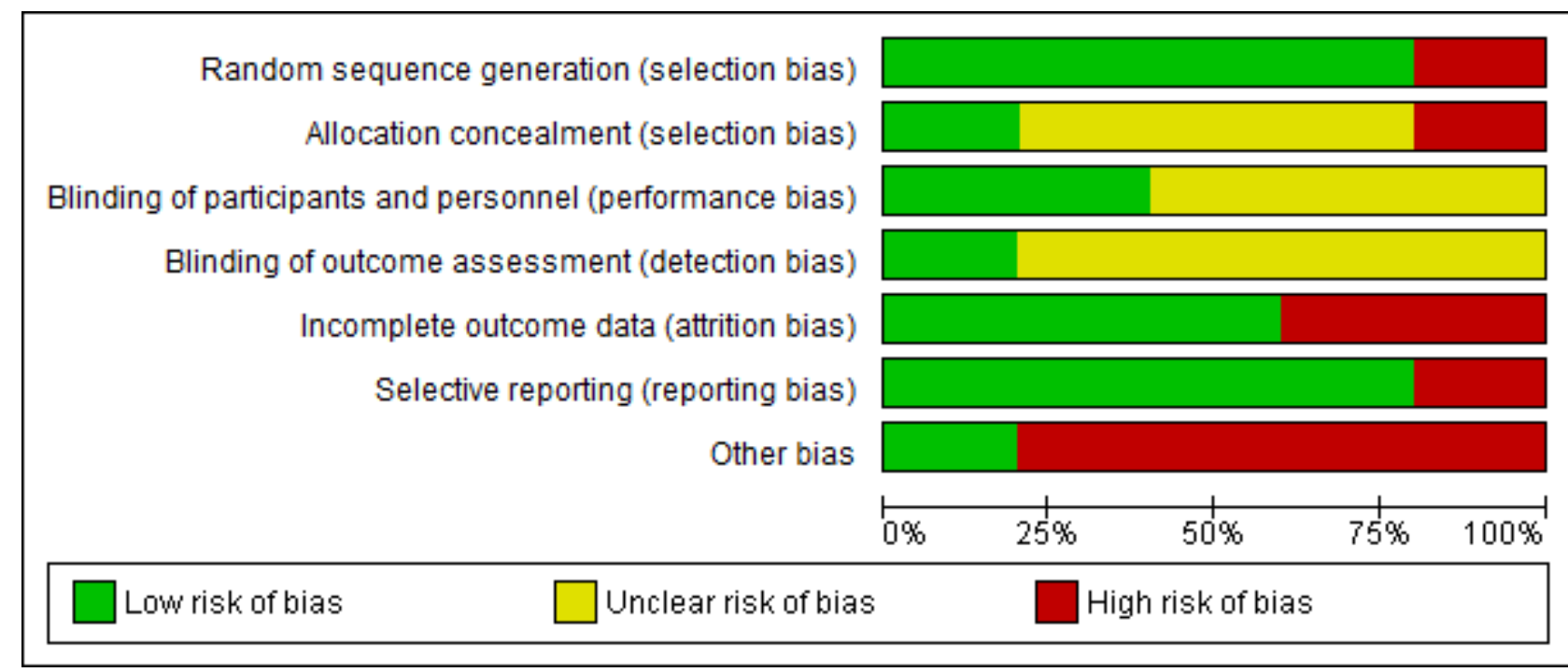

Fig. 3. Risk of bias graph: Review authors' judgements about each risk of bias item presented as percentages across all included studies

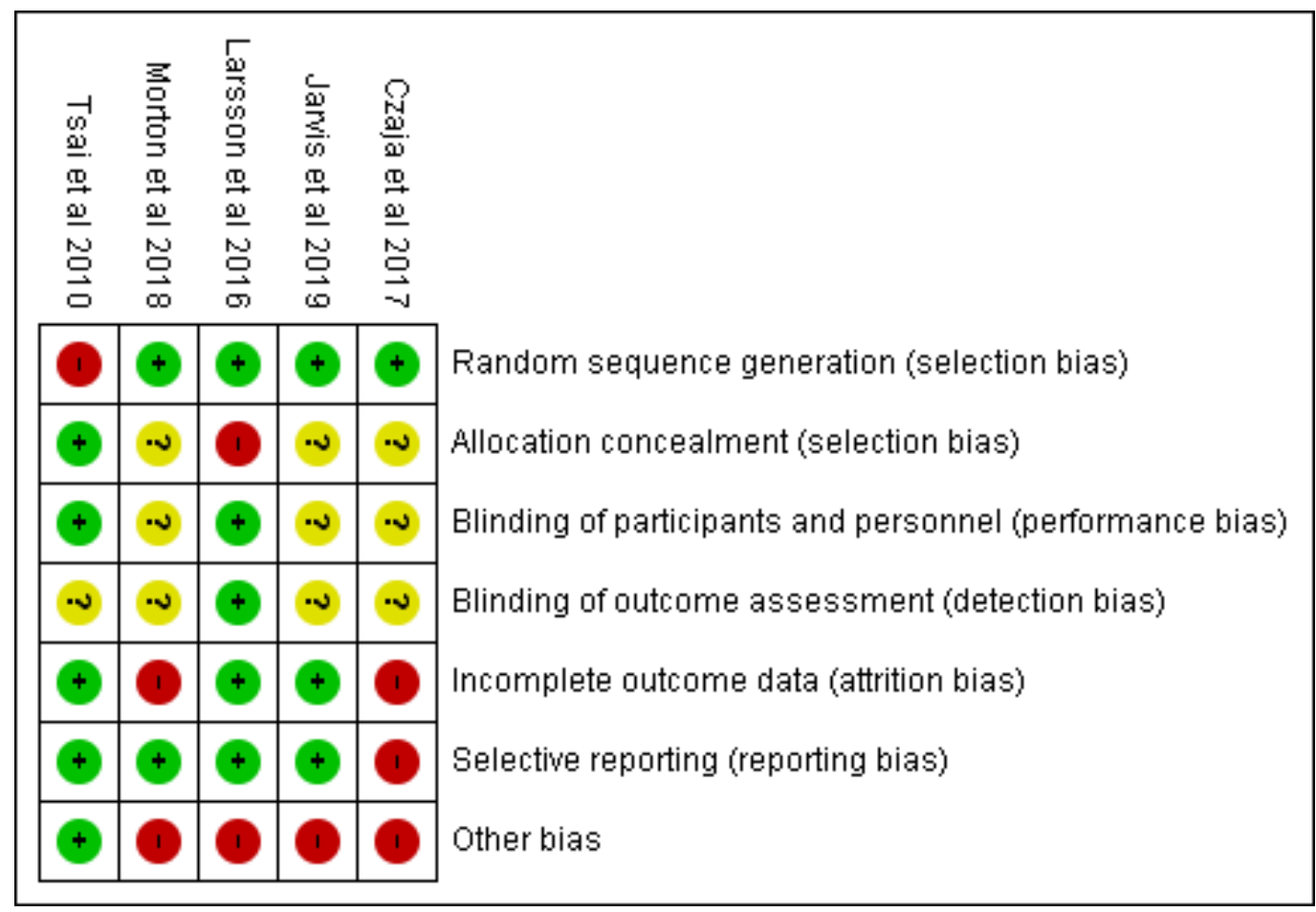

Fig. 4. Risk of bias summary: Review authors' judgements about each risk of bias item for each included study. 
medRxiv preprint doi: https://doi.org/10.1101/2020.08.27.20183012; this version posted September 1, 2020. The copyright holder for this preprint (which was not certified by peer review) is the author/funder, who has granted medRxiv a license to display the preprint in

perpetuity.
It is made available under a CC-BY 4.0 International license .

\subsection{Quality of Evidence}

We assessed the quality of evidence as moderate, very low and moderate in meta-analyses involving three $(44,62,64)$, two $(62,63)$ and two studies $(40,44)$ with follow-up at three, four and six months respectively (Table 3 - GRADE quality of evidence). 
Table 3 GRADE Quality of Evidence

Digital technology interventions compared to usual care or non-digital technology interventions for reducing loneliness in adults (Outcome: Loneliness)

\begin{tabular}{|c|c|c|c|c|c|c|c|c|c|c|c|}
\hline \multicolumn{7}{|c|}{ Certainty assessment } & \multicolumn{5}{|c|}{ Summary of findings } \\
\hline \multirow[b]{2}{*}{$\begin{array}{c}\text { № of } \\
\text { participants } \\
\text { (studies) }\end{array}$} & \multirow[b]{2}{*}{$\begin{array}{c}\text { Risk of } \\
\text { bias }\end{array}$} & \multirow[b]{2}{*}{ Inconsistency } & \multirow[b]{2}{*}{ Indirectness } & \multirow[b]{2}{*}{ Imprecision } & \multirow[b]{2}{*}{$\begin{array}{l}\text { Publication } \\
\text { bias }\end{array}$} & \multirow[b]{2}{*}{$\begin{array}{l}\text { Overall } \\
\text { certainty of } \\
\text { evidence }\end{array}$} & \multicolumn{2}{|c|}{ Study event rates (\%) } & \multirow[b]{2}{*}{$\begin{array}{l}\text { Relative } \\
\text { effect } \\
(95 \% \\
\text { Cl) }\end{array}$} & \multicolumn{2}{|c|}{ Anticipated absolute effects } \\
\hline & & & & & & & $\begin{array}{l}\text { With usual } \\
\text { care or non- } \\
\text { digital } \\
\text { technology } \\
\text { interventions }\end{array}$ & $\begin{array}{l}\text { With Digital } \\
\text { technology } \\
\text { interventions }\end{array}$ & & $\begin{array}{l}\text { Risk with } \\
\text { usual care } \\
\text { or non- } \\
\text { digital } \\
\text { technology } \\
\text { interventions }\end{array}$ & $\begin{array}{c}\text { Risk difference } \\
\text { with Digital } \\
\text { technology } \\
\text { interventions }\end{array}$ \\
\hline
\end{tabular}

Follow up: 3 months

\begin{tabular}{|c|c|c|c|c|c|c|c|c|c|c|c|}
\hline $\begin{array}{c}106 \\
\text { (3 RCTs) }\end{array}$ & $\underset{1, a}{\text { serious }}$ & not serious & not serious & not serious & none & $\begin{array}{c}\oplus \oplus \oplus \bigcirc \\
\text { MODERATE }\end{array}$ & 58 & 48 & - & - & $\begin{array}{c}\text { SMD } 0.02 \text { SD } \\
\text { higher } \\
\text { (0.36 lower to } 0.4 \\
\text { higher) }\end{array}$ \\
\hline
\end{tabular}

\section{Follow up: 4 months}

\begin{tabular}{|c|c|c|c|c|c|c|c|c|c|c|}
\hline $\begin{array}{c}105 \\
\text { (2 RCTs) }\end{array}$ & $\underset{2, \mathrm{a}}{\text { serious }}$ & serious $^{2,3, b}$ & serious $^{2,3, c}$ & serious $^{2,3, d}$ & $\begin{array}{c}\text { publication } \\
\text { bias } \\
\text { strongly } \\
\text { suspected } \\
\text { e }\end{array}$ & $\begin{array}{l}\oplus \bigcirc \bigcirc \bigcirc \\
\text { VERY LOW }\end{array}$ & 48 & 57 & - & $\begin{array}{c}\text { SMD } 1.11 \text { SD } \\
\text { lower } \\
\text { (2.6 lower to } 0.38 \\
\text { higher) }\end{array}$ \\
\hline
\end{tabular}

Follow up: 6 months

\begin{tabular}{|c|c|c|c|c|c|c|c|c|c|c|c|}
\hline $\begin{array}{c}280 \\
\text { (2 RCTs) }\end{array}$ & $\underset{1,4, a}{\text { serious }}$ & not serious & not serious & not serious & none & $\begin{array}{c}\oplus \oplus \oplus \bigcirc \\
\text { MODERATE }\end{array}$ & 132 & 148 & - & - & $\begin{array}{c}\text { SMD } 0.11 \text { SD } \\
\text { lower } \\
\text { (0.54 lower to } \\
0.32 \text { higher) }\end{array}$ \\
\hline
\end{tabular}

Cl: Confidence interval; SMD: Standardised mean difference

Explanations a. Risk of bias possible due to high attrition rate (18.7\%) in Czaja et al (2017) and lack of allocation concealment in a crossover trial ${ }^{44}$. b. Inconsistency possible - High heterogeneity (I squared $=88 \%, P=0.003$ ) observed between studies - differences in participant populations. c. Indirectness possible due to difference in participant populations between studies d. Imprecision possible - Wider and non-overlapping $95 \%$ Cls between studies. e. Publication bias risk possible - Funnel plot shows pooled estimates asymmetrically distributed around the best estimate of effect. 3.6.1.1 References: $1 .{ }^{44}, 2 .{ }^{63}, 3 .{ }^{64}, 4 .^{40}$ 
medRxiv preprint doi: https://doi.org/10.1101/2020.08.27.20183012; this version posted September 1, 2020. The copyright holder for this

preprint (which was not certified by peer review) is the author/funder, who has granted medRxiv a license to display the preprint in perpetuity.

It is made available under a CC-BY 4.0 International license .

\section{DISCUSSION}

To provide the answer whether digital technology is effective in reducing loneliness in adults, we appraised peer reviewed empirical research involving the application of DTIs in adults with loneliness. Our systematic review includes a narrative summary of six studies, which reported reduction in loneliness in the intervention groups at the follow-up compared to the baseline (Table 2). However, our meta-analyses of five clinical trials with follow-up measurements at three, four and six months showed no statistically significant pooled effect estimates as SMDs - the preferred method for summarizing effects on continuous outcomes such as loneliness. Although not statistically significant, the summary effect size at four months follow-up was better compared to the effect size at three and six months follow-up (Fig. 2).

Our meta-analysis also revealed that Cls of the summary effects of two studies i.e. (44). and (64) were very wide and the SMDs from these studies were more in favour of control group rather than the intervention group (Fig. 2). Thus, the wide widths of Cls of the summary effects in these studies leave uncertainty about the beneficial effect or otherwise of DTI on measures of loneliness.

Overall, findings of our meta-analysis provide insufficient evidence to make conclusions that DTIs are effective in reducing loneliness in (older) adults.

\subsection{Summary of evidence}

The quality of evidence of included studies was very low to moderate (Table 3) and there was a high heterogeneity between studies $(62,63)$. All included studies had a high proportion of female participants. Most notably, the total number of participants was low (44, 62 ) and the sample sizes were reduced further due to a high attrition rate in some studies $(40,63)$. The types and methods of DTIs varied between studies, which were conducted in diverse settings and different countries (Tables 1-2). These factors could have contributed in pooled estimates being not statistically significant.

There a few published meta-analyses on technological interventions for tackling loneliness covering literature published up to 2009 (25) and 2011 (28) whereas our review and metaanalysis includes the latest evidence published between 1 January 2010 and 31 July 2019. We did not replicate the findings of earlier meta-analyses that reported evidence suggesting technological interventions resulting in decreased loneliness $(25,28)$. A meta-analysis by (28) reported statistically significant evidence suggesting internet and computers reduce loneliness. However, (28) focused on older adults with depression and included the Internet and computers only as technological interventions whereas we included all types of digital technology interventions and adults of all age groups ( $\geq 18$ years). In addition, studies $(n=5)$ included in the meta-analysis by (28) had different follow-up periods (3-6 months) but they did not report which follow-up measurements they included in their meta-analysis. We ran different meta-analyses for measurements at different follow-up periods, i.e. three, four and six months, as suggested by the Cochrane guidelines (47).

In addition, a meta-analysis by Masi et al., 2011 also reported that technological interventions reduce loneliness, which was more in pre-post studies and non-randomised studies compared to RCTs. However, they included studies with technology and non- 
medRxiv preprint doi: https://doi.org/10.1101/2020.08.27.20183012; this version posted September 1,2020 . The copyright holder for this preprint (which was not certified by peer review) is the author/funder, who has granted medRxiv a license to display the preprint in perpetuity.

It is made available under a CC-BY 4.0 International license .

technology based interventions (25) whereas we focused on studies with DTIs only. Masi et al., 2011 did not report how they used measurements at different follow-up periods while we avoided combining follow-up measurements at different times as suggested (47). Nonetheless, Masi et al concluded that the technology is yet to be capitalised on for loneliness (25), which is evident from our findings that show that DTIs do not reduce loneliness, especially in adults.

Interestingly, our findings provide new insights about loneliness in older adults. Despite our inclusion criteria of age (18 years and above), the selected studies more commonly involved older people (averagely 70+ years old) and there was no statistically significant reduction in loneliness. Our findings might suggest that DTIs do not reduce loneliness in adults, which is opposite to a commonly held view that digital technology can solve the problem of loneliness, especially in older people. However, the digital technologies are tools and means to social connectedness, which may help in lessening loneliness for a little while because the effects of DTIs are short-lived and do not last beyond six months of the intervention (66). In addition, digital technologies might provide digital social connection but in fact they reduce social connectedness in real life (67). Digital technologies are only tools that extend opportunities for connecting with others but do not provide real human interaction (68) and cannot replace human contact (40).

\subsection{Limitations}

Limitations of our systematic reviews include a small number of studies $(n=6)$ with heterogeneous sets of results, the selection of English language peer reviewed literature published from January 1, 2010 to July 31, 2019 and the intervention duration minimum of three months, which could have resulted in the inclusion of a low number of studies and possible exclusion of potential studies that would have provided useful evidence.

In addition, we could not run sub-group and meta-regression analyses due to very limited number of studies and lack of data on loneliness by participants' demographic characteristics. In addition, our review might be narrow because we excluded some studies $(39,69-75)$, which met the technology criterion such as the use of robots, sensors, digital speakers and apps but failed to meet other selection criteria. Thus, our meta-analysis was limited to studies mostly about social interactions using digital tools.

Moreover, another limitation could be using a meta-analysis based on only the follow-up data. For example, study by Tsai et al., 2010 in the 3 months follow-up meta-analysis had an SMD of 0.06 with a $\mathrm{Cl}-0.8$ to $+0.65(\mathrm{p}=0.03)$ (Fig. 2$)$, which may suggest that these studies may have had higher power to show a difference compared to baseline loneliness.

As recommendations for future research, we suggest that researchers involved in trials may agree on a common measure of loneliness and consider reporting of results in a standardised way that would allow pooling of baseline-adjusted estimates of the treatment effect rather than differences in follow-up means.

\section{CONCLUSIONS}

We did not find sufficient evidence supporting the effectiveness of digital technology intervention to reduce loneliness in (older) adults. Our findings may suggest further research 
medRxiv preprint doi: https://doi.org/10.1101/2020.08.27.20183012; this version posted September 1 , 2020. The copyright holder for this preprint (which was not certified by peer review) is the author/funder, who has granted medRxiv a license to display the preprint in perpetuity.

It is made available under a CC-BY 4.0 International license.

involving RCTs (44) with larger sample sizes with longer duration of interventions and followup measurement periods. Future research may apply inclusive research designs using a combination of digital applications including robots, sensors and social connecting applications by involving adults in the age group 45-65 years as this segment of the population is more likely to be more technology savvy and digital interventions might be more effective. Future research might also target ethnic, racial, and sexual orientation minority communities where loneliness is common (11).

Previous presentation: A poster on the preliminary findings of our systematic review presented at the 12th European Public Health Conference, 20-23 November 2019, Marseille, France. The abstract of the poster was published in the European Journal of Public Health, Vol. 29, Supplement 4, 2019

Disclosure of Competing Interests and Financial Support: Authors declare no competing interests.

Funding: This research was funded/supported by the National Institute for Health Research (NIHR) Oxford Biomedical Research Centre (Research Grant No. IS-BRC-1215-20008). The views expressed are those of the author(s) and not necessarily those of the NHS, the NIHR or the Department of Health.

Acknowledgements: The authors acknowledge support from Liz Callow of Bodleian Health Care Libraries, University of Oxford for running literature searches. We gratefully thank to Dr Alexandra Farrow, Brunel University London for checking the manuscript.

\section{REFERNCES}

1. Lindsay EK, Young S, Brown KW, et al.: Mindfulness training reduces loneliness and increases social contact in a randomized controlled trial. Proc Natl Acad Sci U S A 2019; 116:3488-3493

2. Galea S, Merchant RM, Lurie N: The Mental Health Consequences of COVID-19 and Physical Distancing: The Need for Prevention and Early Intervention [Internet]. JAMA Intern Med 2020; 180:817-818[cited 2020 Apr 27] Available from: https://doi.org/10.1001/jamainternmed.2020.1562

3. Holmes EA, O'Connor RC, Perry VH, et al.: Multidisciplinary research priorities for the COVID19 pandemic: a call for action for mental health science [Internet]. Lancet Psychiat 2020; Online first[cited 2020 Apr 27] Available from: https://doi.org/10.1016/S2215-0366(20)30168-1

4. World Health Organization: Mental health and COVID-19 outbreak. Technical Guidance [Internet]2020; Available from: http://www.euro.who.int/en/health-topics/healthemergencies/coronavirus-covid-19/novel-coronavirus-2019-ncov-technicalguidance/coronavirus-disease-covid-19-outbreak-technical-guidance-europe/mental-health-andcovid-19

5. The Economist: Loneliness is pervasive and rising, particularly among the young - Daily chart [Internet]. Graphic detail 2018; [cited 2019 May 3] Available from: https://www.economist.com/graphic-detail/2018/08/31/loneliness-is-pervasive-and-risingparticularly-among-the-young

6. Relationships Australia: An epidemic of loneliness 2001-2017 [Internet]2017; [cited 2019 May 3] Available from: https://www.relationships.org.au/what-we-do/research/an-epidemic-ofloneliness-2001-2017/view

7. van den Broek T: Gender differences in the correlates of loneliness among Japanese persons aged 50-70. Australas J Ageing 2017; 36:234-237 
medRxiv preprint doi: https://doi.org/10.1101/2020.08.27.20183012; this version posted September 1 , 2020. The copyright holder for this preprint (which was not certified by peer review) is the author/funder, who has granted medRxiv a license to display the preprint in

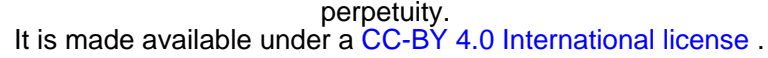

8. Victor CR, Bowling A: A longitudinal analysis of loneliness among older people in Great Britain. J Psychol 2012; 146:313-331

9. Murthy V: Work and the loneliness epidemic [Internet]. Harv Bus Rev 2017; 9Available from: https://hbr.org/cover-story/2017/09/work-and-the-loneliness-epidemic

10. Cohen-Mansfield J, Perach R: Interventions for alleviating loneliness among older persons: a critical review. Am J Health Promot 2015; 29:e109-e125

11. Jeste DV, Lee EE, Cacioppo S: Battling the Modern Behavioral Epidemic of Loneliness: Suggestions for Research and Interventions [Internet]. JAMA Psychiatry 2020; [cited 2020 Apr 28] Available from: https://doi.org/10.1001/jamapsychiatry.2020.0027

12. Gao Q, Prina $M$, Prince $M$, et al.: Loneliness among older adults in Latin America, China, and India: prevalence, correlates and association with mortality [Internet]. Square Research (Preprint) 2020; 19700/v1Available from: http://europepmc.org/abstract/PPR/PPR134343

13. Okwaraji FE, Obiechina KI, Onyebueke GC, et al.: Loneliness, life satisfaction and psychological distress among out-of-school adolescents in a Nigerian urban city. Psychol Health Med 2018; 23:1106-1112

14. The Scottish Government: A Connected Scotland: our strategy for tackling social isolation and loneliness and building stronger social connections [Internet]2018; [cited 2019 May 3] Available from: https://www.gov.scot/publications/connected-scotland-strategy-tackling-social-isolationloneliness-building-stronger-social-connections/pages/6/

15. Qualter P: Loneliness in children and adolescents: What do schools and teachers need to know and how can they help? Pastor Care Educ 2003; 21:10-18

16. Cohen-Mansfield J, Hazan H, Lerman Y, et al.: Correlates and predictors of loneliness in olderadults: a review of quantitative results informed by qualitative insights. Int Psychogeriatr 2016; 28:557-576

17. Deckx L, van den Akker M, Buntinx F: Risk factors for loneliness in patients with cancer: a systematic literature review and meta-analysis. Eur J Oncol Nurs 2014; 18:466-477

18. Chen Y, Hicks A, While AE: Loneliness and social support of older people in China: a systematic literature review. Health Soc Care Community 2014; 22:113-123

19. Rico-Uribe LA, Caballero FF, Martín-María N, et al.: Association of loneliness with all-cause mortality: A meta-analysis [Internet]. PLOS ONE 2018; 13:e0190033Available from: https://doi.org/10.1371/journal.pone.0190033

20. Holt-Lunstad J: The potential public health relevance of social isolation and loneliness: Prevalence, epidemiology, and risk factors. Public Policy Aging Rep 2017; 27:127-130

21. Holt-Lunstad J, Smith TB, Baker M, et al.: Loneliness and social isolation as risk factors for mortality: a meta-analytic review. Perspectives on psychological science 2015; 10:227-237

22. Deckx $L$, van den Akker $M$, Buntinx $F$, et al.: A systematic literature review on the association between loneliness and coping strategies. Psychol Health Med 2018; 23:899-916

23. Lara E, Martín-María N, De la Torre-Luque A, et al.: Does loneliness contribute to mild cognitive impairment and dementia? A systematic review and meta-analysis of longitudinal studies [Internet]. Ageing Res Rev 2019; 52:7-16Available from: http://www.sciencedirect.com/science/article/pii/S1568163718302472

24. Steptoe A, Shankar A, Demakakos $P$, et al.: Social isolation, loneliness, and all-cause mortality in older men and women. Proc Natl Acad Sci U S A 2013; 110:5797-5801 
medRxiv preprint doi: https://doi.org/10.1101/2020.08.27.20183012; this version posted September 1 , 2020. The copyright holder for this preprint (which was not certified by peer review) is the author/funder, who has granted medRxiv a license to display the preprint in perpetuity.

It is made available under a CC-BY 4.0 International license.

25. Masi CM, Chen H-Y, Hawkley LC, et al.: A meta-analysis of interventions to reduce loneliness. Pers Soc Psychol Rev 2011; 15:219-266

26. Pu L, Moyle W, Jones $\mathrm{C}$, et al.: The effectiveness of social robots for older adults: A systematic review and meta-analysis of randomized controlled studies. Gerontologist 2019; 59:e37-e51

27. Medical Advisory Secretariat: Social isolation in community-dwelling seniors: an evidence-based analysis. Ont Health Technol Assess Ser 2008; 8:1-49

28. Choi M, Kong S, Jung D: Computer and internet interventions for loneliness and depression in older adults: a meta-analysis. Healthc Inform Res 2012; 18:191-198

29. Morris ME, Adair B, Miller K, et al.: Smart-home technologies to assist older people to live well at home. J Aging Health 2013; 1:1-9

30. Morris ME, Adair B, Ozanne E, et al.: Smart technologies to enhance social connectedness in older people who live at home. Australas J Ageing 2014; 33:142-152

31. Pearce AJ, Adair B, Miller K, et al.: Robotics to enable older adults to remain living at home. $\mathrm{J}$ Aging Res 2012; 2012:538169

32. Hagan R, Manktelow R, Taylor BJ, et al.: Reducing loneliness amongst older people: a systematic search and narrative review. Aging Ment Health 2014; 18:683-693

33. Miller KJ, Adair BS, Pearce AJ, et al.: Effectiveness and feasibility of virtual reality and gaming system use at home by older adults for enabling physical activity to improve health-related domains: a systematic review. Age Ageing 2014; 43:188-195

34. Shah SGS, Nogueras D, Woerden $\mathrm{H}$ van, et al.: Effectiveness of digital technology interventions to reduce loneliness in adults: a protocol for a systematic review and meta-analysis [Internet]. BMJ Open 2019; 9 :e032455[cited 2019 Dec 20] Available from: https://bmjopen.bmj.com/content/9/9/e032455

35. Shah SGS, Nogueras D, van Woerden $\mathrm{H}$, et al.: The effectiveness of digital technology interventions to reduce loneliness in adult people: a systematic review and meta-analysis. PROSPERO 2019 CRD42019131524 [Internet]2019; [cited 2019 Jun 10] Available from: https://www.crd.york.ac.uk/PROSPERO/display_record.php?RecordID=131524

36. Harmon J: What are digital technologies? [Internet]2018; [cited 2019 Jun 6] Available from: https://www.quora.com/What-are-digital-technologies

37. Hemberg J, Santamäki Fischer R: A Window Toward the World: Older Adults' Experiences of Becoming in Health and Developing as Human Beings Through Interacting With Others Using Real Video Communication [Internet]. Holist Nurs Pract 2018; 32:90-97Available from: https://journals.Iww.com/hnpjournal/Fulltext/2018/03000/A_Window_Toward_the_World_Older _Adults_.6.aspx

38. Gao Q, Ebert D, Chen X, et al.: Design of a Mobile Social Community Platform for Older Chinese People in Urban Areas [Internet]. Hum Factors Ergon Manuf 2015; 25:66-89[cited 2020 Jan 2] Available from: https://doi.org/10.1002/hfm.20523

39. Austin J, Dodge HH, Riley T, et al.: A Smart-Home System to Unobtrusively and Continuously Assess Loneliness in Older Adults. IEEE J Transl Eng Health Med 2016; 4:Art. No. 2800311

40. Czaja SJ, Boot WR, Charness N, et al.: Improving Social Support for Older Adults Through Technology: Findings From the PRISM Randomized Controlled Trial [Internet]. Gerontologist 2018; 58:467-477[cited 2019 Dec 27] Available from: https://doi.org/10.1093/geront/gnw249 
medRxiv preprint doi: https://doi.org/10.1101/2020.08.27.20183012; this version posted September 1 , 2020. The copyright holder for this preprint (which was not certified by peer review) is the author/funder, who has granted medRxiv a license to display the preprint in perpetuity.

It is made available under a CC-BY 4.0 International license.

41. Shamseer $L$, Moher D, Clarke M, et al.: Preferred reporting items for systematic review and meta-analysis protocols (PRISMA-P) 2015: elaboration and explanation [Internet]. BMJ 2015; 349:g7647Available from: http://www.bmj.com/content/349/bmj.g7647.abstract

42. Munn Z, Tufanaru C, Aromataris E: JBl's systematic reviews: data extraction and synthesis. Am J Nurs 2014; 114:49-54

43. Munn Z, Stern C, Aromataris E, et al.: What kind of systematic review should I conduct? A proposed typology and guidance for systematic reviewers in the medical and health sciences [Internet]. BMC Med Res Methodol 2018; 18:5-5Available from: https://www.ncbi.nlm.nih.gov/pubmed/29316881

44. Larsson E, Padyab M, Larsson-Lund M, et al.: Effects of a social internet-based intervention programme for older adults: An explorative randomised crossover study [Internet]. $\mathrm{Br} \mathrm{J}$ Occup Ther 2016; 79:629-636[cited 2019 Dec 27] Available from: https://doi.org/10.1177/0308022616641701

45. Larsson E, Nilsson I, Larsson Lund M: Participation in social internet-based activities: Five seniors' intervention processes [Internet]. Scand J Occup Ther 2013; 20:471-480Available from: https://doi.org/10.3109/11038128.2013.839001

46. Tufanaru C, Munn Z, Aromataris E, et al.: Chapter 3: Systematic reviews of effectiveness [Internet], inAromataris E, Munn Z, editorsJoanna Briggs Institute Reviewer's Manual. Adelaide, Joanna Briggs Institute (JBI), 2017[cited 2019 May 3] Available from: Available from: https://wiki.joannabriggs.org/display/MANUAL/Chapter+3\%3A+Systematic+reviews+of+effective ness

47. Higgins JP, Green S: Cochrane Handbook for Systematic Reviews of Interventions [Internet]. Version 5.1.0 [updated March 2011]. The Cochrane Collaboration, 2011[cited 2019 May 13] Available from: https://handbook-5-1.cochrane.org/front_page.htm

48. Russell DW: UCLA Loneliness Scale (Version 3): Reliability, Validity, and Factor Structure [Internet]. J Pers Assess 1996; 66:20-40Available from: https://doi.org/10.1207/s15327752jpa6601_2

49. Russell D, Peplau LA, Cutrona CE: The revised UCLA Loneliness Scale: Concurrent and discriminant validity evidence. J Pers Soc Psychol 1980; 39:472-480

50. de Jong Gierveld J, Tilburg TV: A 6-item scale for overall, emotional, and social loneliness: Confirmatory tests on survey data. Res Aging 2006; 28:582-598

51. The Nordic Cochrane Centre, The Cochrane Collaboration: Review Manager (RevMan) Version 5.3.5 [Internet]. Copenhagen, 2014[cited 2019 Jul 19] Available from: https://community.cochrane.org/help/tools-and-software/revman-5

52. Borenstein M, Hedges LV, Higgins JP, et al.: Introduction to meta-analysis. Chichester, John Wiley \& Sons, 2011

53. Borenstein M, Hedges LV, Higgins JP, et al.: A basic introduction to fixed-effect and randomeffects models for meta-analysis. Res Synth Methods 2010; 1:97-111

54. da Costa BR, Jüni P: Systematic reviews and meta-analyses of randomized trials: principles and pitfalls [Internet]. Eur Heart J 2014; 35:3336-3345[cited 2019 Jul 22] Available from: https://doi.org/10.1093/eurheartj/ehu424

55. Eccleston C, Fisher E, Craig L, et al.: Psychological therapies (Internet-delivered) for the management of chronic pain in adults [Internet]. Cochrane Database Syst Rev 2014; 2014:CD010152-CD010152Available from: https://pubmed.ncbi.nlm.nih.gov/24574082 
medRxiv preprint doi: https://doi.org/10.1101/2020.08.27.20183012; this version posted September 1 , 2020. The copyright holder for this preprint (which was not certified by peer review) is the author/funder, who has granted medRxiv a license to display the preprint in perpetuity.

It is made available under a CC-BY 4.0 International license .

56. van der Heide LA, Willems CG, Spreeuwenberg MD, et al.: Implementation of CareTV in care for the elderly: the effects on feelings of loneliness and safety and future challenges. Technol Disabil 2012; 24:283-291

57. Grimshaw J, Campbell M, Eccles M, et al.: Experimental and quasi-experimental designs for evaluating guideline implementation strategies [Internet]. Fam Pract 2000; 17:S11-S16[cited 2020 Jan 4] Available from: https://doi.org/10.1093/fampra/17.suppl_1.S11

58. Guyatt GH, Oxman AD, Sultan S, et al.: GRADE guidelines: 9. Rating up the quality of evidence [Internet]. J Clin Epidemiol 2011; 64:1311-1316[cited 2019 Jun 10] Available from: https://doi.org/10.1016/j.jclinepi.2011.06.004

59. Sedgwick P: Meta-analyses: how to read a funnel plot [Internet]. BMJ 2013; 346:f1342Available from: http://www.bmj.com/content/346/bmj.f1342.abstract

60. Higgins JPT, Thompson SG: Quantifying heterogeneity in a meta-analysis [Internet]. Stat Med 2002; 21:1539-1558[cited 2019 May 13] Available from: https://doi.org/10.1002/sim.1186

61. Higgins JPT, Thompson SG, Deeks JJ, et al.: Measuring inconsistency in meta-analyses [Internet]. BMJ 2003; 327:557Available from: http://www.bmj.com/content/327/7414/557.abstract

62. Jarvis MA, Padmanabhanunni A, Chipps J: An Evaluation of a Low-Intensity Cognitive Behavioral Therapy mHealth-Supported Intervention to Reduce Loneliness in Older People [Internet]. Int J Environ Res Public Health 2019; 16:1305Available from: https://www.ncbi.nlm.nih.gov/pubmed/30979042

63. Morton TA, Wilson N, Haslam C, et al.: Activating and Guiding the Engagement of Seniors With Online Social Networking: Experimental Findings From the AGES 2.0 Project [Internet]. J Aging Health 2018; 30:27-51[cited 2019 Dec 27] Available from: https://doi.org/10.1177/0898264316664440

64. Tsai $\mathrm{H}-\mathrm{H}$, Tsai $\mathrm{Y}-\mathrm{F}$, Wang $\mathrm{H}-\mathrm{H}$, et al.: Videoconference program enhances social support, loneliness, and depressive status of elderly nursing home residents [Internet]. Aging Ment Health 2010; 14:947-954Available from: https://doi.org/10.1080/13607863.2010.501057

65. De Jong Gierveld J, Van Tilburg T: The De Jong Gierveld short scales for emotional and social loneliness: tested on data from 7 countries in the UN generations and gender surveys [Internet]. Eur J Ageing 2010; 7:121-130Available from: https://doi.org/10.1007/s10433-010-0144-6

66. Chen Y-RR, Schulz PJ: The Effect of Information Communication Technology Interventions on Reducing Social Isolation in the Elderly: A Systematic Review [Internet]. J Med Internet Res 2016; 18:e18-e18Available from: https://pubmed.ncbi.nlm.nih.gov/26822073

67. Kushlev K, Proulx JDE, Dunn EW: Digitally connected, socially disconnected: The effects of relying on technology rather than other people [Internet]. Comput Human Behav 2017; 76:6874Available from: http://www.sciencedirect.com/science/article/pii/S0747563217304132

68. Anderson GO, Thayer CE: Loneliness and Social Connections: A National Survey of Adults 45 and Older [Internet]. Washington, DC., AARP Foundation, 2018Available from:

https://www.aarp.org/content/dam/aarp/research/surveys_statistics/life-leisure/2018/lonelinesssocial-connections-2018.doi.10.26419-2Fres.00246.001.pdf

69. Ballantyne A, Trenwith L, Zubrinich S, et al.: 'I feel less lonely': what older people say about participating in a social networking website [Internet]. Qual Ageing Older Adults 2010; 11:2535[cited 2020 Aug 3] Available from: https://doi.org/10.5042/qiaoa.2010.0526

70. Kahlbaugh PE, Sperandio AJ, Carlson AL, et al.: Effects of Playing Wii on Well-Being in the Elderly: Physical Activity, Loneliness, and Mood [Internet]. Act Adapt Aging 2011; 35:331344Available from: https://doi.org/10.1080/01924788.2011.625218 
medRxiv preprint doi: https://doi.org/10.1101/2020.08.27.20183012; this version posted September 1, 2020. The copyright holder for this preprint (which was not certified by peer review) is the author/funder, who has granted medRxiv a license to display the preprint in perpetuity.

It is made available under a CC-BY 4.0 International license.

71. Blažun H, Saranto K, Rissanen S: Impact of computer training courses on reduction of loneliness of older people in Finland and Slovenia [Internet]. Comput Hum Behav 2012; 28:1202-1212Available from:

http://www.sciencedirect.com/science/article/pii/S0747563212000350

72. Blusi M, Asplund K, Jong M: Older family carers in rural areas: experiences from using caregiver support services based on Information and Communication Technology (ICT) [Internet]. Eur J Ageing 2013; 10:191-199Available from: https://pubmed.ncbi.nlm.nih.gov/28804294

73. Chopik WJ: The Benefits of Social Technology Use Among Older Adults Are Mediated by Reduced Loneliness [Internet]. Cyberpsychol Behav Soc Netw 2016; 19:551-556[cited 2020 Aug 3] Available from: https://doi.org/10.1089/cyber.2016.0151

74. Siniscarco MT, Love-Williams C, Burnett-Wolle S: Video Conferencing: An Intervention for Emotional Loneliness in Long-Term Care [Internet]. Act Adapt Aging 2017; 41:316-329Available from: https://doi.org/10.1080/01924788.2017.1326763

75. Neves BB, Franz R, Judges R, et al.: Can Digital Technology Enhance Social Connectedness Among Older Adults? A Feasibility Study [Internet]. J Appl Gerontol 2019; 38:49-72[cited 2019 May 2] Available from: https://doi.org/10.1177/0733464817741369 
Appendix 1. Literature searches

\begin{tabular}{|c|c|c|c|c|c|}
\hline \multirow[t]{2}{*}{ Search Query } & \multicolumn{5}{|c|}{ DATABASES SEARCHED } \\
\hline & PubMed & EMBASE & Medline & CINAHL & Web of Science \\
\hline Subject Headings & & (via OVID) & (via OVID) & (via EBSCOHOST) & \\
\hline Loneliness & Yes & Yes & Yes & Yes & No subject headings \\
\hline Technology & No & No & No & Yes & No subject headings \\
\hline Robotics & Yes & Yes & Yes & Yes & No subject headings \\
\hline Internet & Yes & Yes & Yes & Yes & No subject headings \\
\hline Social Media & Yes & Yes & Yes & Yes & No subject headings \\
\hline Telephone & Yes & Yes & Yes & Yes & No subject headings \\
\hline Smartphone & Yes & Yes & Yes & Yes & No subject headings \\
\hline Computer & Yes & Yes & Yes & Yes & No subject headings \\
\hline Computer handheld & Yes & No & Yes & Yes & No subject headings \\
\hline Personal digital assistant & No & Yes & No & No & No subject headings \\
\hline Web (world Wide Web) & No & No & No & Yes & No subject headings \\
\hline \multicolumn{6}{|l|}{ Phrases / text } \\
\hline $\begin{array}{l}\text { Loneliness OR lonely OR social } \\
\text { isolation [Title/Abstract] }\end{array}$ & Yes & Yes & Yes & Yes & $\begin{array}{l}\text { Searched as topics (includes: } \\
\text { Title, Abstract, Author Keywords, } \\
\text { Keywords Plus) }\end{array}$ \\
\hline \multicolumn{6}{|r|}{ 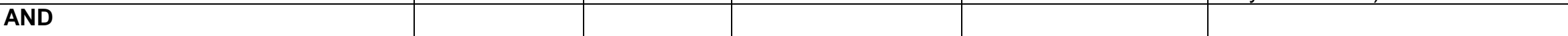 } \\
\hline $\begin{array}{l}\text { Digital }^{*} \text { OR technolog* } \text { OR sensor }^{*} \text { OR } \\
\text { robot* OR internet OR social media } \\
\text { OR smartphone* OR smart phone* } \\
\text { OR telephone* OR phone OR online } \\
\text { OR ipad* OR computer* OR } \\
\text { electronic* OR Web [Title/Abstract] }\end{array}$ & Yes & Yes & Yes & Yes & $\begin{array}{l}\text { digital }^{*} \text { OR technolog* OR } \\
\text { sensor }^{*} \text { OR robot }{ }^{*} \text { OR internet } \\
\text { OR social media OR smartphone* } \\
\text { OR online OR ipad* OR } \\
\text { computer }^{*} \text { OR electronic } \\
\text { Web } \text { [Searched as topics] } \\
\end{array}$ \\
\hline \multicolumn{6}{|l|}{ Filters } \\
\hline Publication years: $2010-2019$ & Yes & Yes & Yes & Yes & Yes \\
\hline Language: English & Yes & Yes & Yes & Yes & Yes \\
\hline Species: Humans & Yes & Yes & Yes & Yes & $\mathrm{N} / \mathrm{A}$ \\
\hline
\end{tabular}




\begin{tabular}{|c|c|c|c|c|c|}
\hline Document types: & $\begin{array}{l}\text { Clinical Trial, } \\
\text { Controlled } \\
\text { Clinical Trial, } \\
\text { Evaluation } \\
\text { Studies, } \\
\text { Journal } \\
\text { Article, } \\
\text { Meta- } \\
\text { Analysis, } \\
\text { Observational } \\
\text { Study, } \\
\text { Randomized } \\
\text { Controlled } \\
\text { Trial, } \\
\text { Review, } \\
\text { Systematic } \\
\text { Reviews }\end{array}$ & Trials (all) & $\begin{array}{l}\text { Clinical Trial, } \\
\text { Evaluation Studies } \\
\text { Journal Article } \\
\text { Meta-Analysis } \\
\text { Observational } \\
\text { Study } \\
\text { Review } \\
\text { Systematic } \\
\text { Reviews }\end{array}$ & $\begin{array}{l}\text { Clinical Trial, } \\
\text { Journal Article, } \\
\text { Meta-Analysis, } \\
\text { Randomized } \\
\text { Controlled Trial, } \\
\text { Research, Review } \\
\text { Systematic Review }\end{array}$ & Articles, Reviews \\
\hline
\end{tabular}

\section{PUBMED SEARCHES}

(("Loneliness"[Mesh]) OR (((loneliness[Title/Abstract]) OR lonely[Title/Abstract]) OR social isolation[Title/Abstract])) AND (("Robotics"[Mesh]) OR ("Internet"[Mesh]) OR ("Social Media"[Mesh]) OR ("Smartphone"[Mesh]) OR ("Telephone"[Mesh]) OR ("Computers, Handheld"[Mesh]) OR ("Computers"[Mesh]) OR ((()((()((((digital*[Title/Abstract]) OR technolog*[Title/Abstract]) OR sensor*[Title/Abstract]) OR robot*[Title/Abstract]) OR internet[Title/Abstract]) OR social media[Title/Abstract]) OR phone*[Title/Abstract]) OR telephone*[Title/Abstract]) OR online[Title/Abstract]) OR ipad*[Title/Abstract]) OR computer*[Title/Abstract]) OR electronic*[Title/Abstract]) OR web[Title/Abstract]))

("Loneliness"[Mesh] OR ((loneliness[Title/Abstract] OR lonely[Title/Abstract]) OR social isolation[Title/Abstract])) AND ("Robotics"[Mesh] OR "Internet"[Mesh] OR "Social Media"[Mesh] OR "Smartphone"[Mesh] OR "Telephone"[Mesh] OR "Computers, Handheld"[Mesh] OR "Computers"[Mesh] OR $((()(()((()($ digital[Title/Abstract] OR digital'[Title/Abstract] OR digital"[Title/Abstract] OR digital's[Title/Abstract] OR digital1[Title/Abstract] OR digitala[Title/Abstract] OR digitalassets[Title/Abstract] OR digitalb[Title/Abstract] OR digitalcell[Title/Abstract] OR digitalcellsorter[Title/Abstract] OR digitalcommunication[Title/Abstract] OR digitaldiagnost[Title/Abstract] OR digitaldlsorter[Title/Abstract] OR digitaldna[Title/Abstract] OR digitale[Title/Abstract] OR digitaleae[Title/Abstract] OR digitalel[Title/Abstract] OR digitalemia[Title/Abstract] OR digitalemia's[Title/Abstract] OR digitalemic[Title/Abstract] OR digitalen[Title/Abstract] OR digitaler[Title/Abstract] OR digitalera[Title/Abstract] OR digitales[Title/Abstract] OR digitalface[Title/Abstract] OR digitalfiltering[Title/Abstract] OR digitalfishlibrary[Title/Abstract] OR digitalgia[Title/Abstract] OR digitalglobe[Title/Abstract] OR digitalhealth[Title/Abstract] OR digitalhealtheurope[Title/Abstract] OR digitalhealthscore[Title/Abstract] OR digitalhub[Title/Abstract] OR digitalhub's[Title/Abstract] OR digitali[Title/Abstract] OR digitalia[Title/Abstract] OR digitalic[Title/Abstract] OR digitalica[Title/Abstract] OR digitalics[Title/Abstract] OR digitalideae[Title/Abstract] OR digitalidis[Title/Abstract] OR digitalin[Title/Abstract] OR digitalinaemia[Title/Abstract] OR digitaline[Title/Abstract] OR digitalins[Title/Abstract] OR 
digitalinum[Title/Abstract] OR digitalis[Title/Abstract] OR digitalis'[Title/Abstract] OR digitalis's[Title/Abstract] OR digitalisation[Title/Abstract] OR digitalisconcentration[Title/Abstract] OR digitalisconcentrations[Title/Abstract] OR digitalise[Title/Abstract] OR digitalised[Title/Abstract] OR digitalisglycoside[Title/Abstract] OR digitalisglycosides[Title/Abstract] OR digitalisierten[Title/Abstract] OR digitalisierung[Title/Abstract] OR digitalising[Title/Abstract] OR digitalisintoxication[Title/Abstract] OR digitalisintoxications[Title/Abstract] OR digitalisize[Title/Abstract] OR digitalislike[Title/Abstract] OR digitalism[Title/Abstract] OR digitalispreparations[Title/Abstract] OR digitalisreceptor[Title/Abstract] OR digitality[Title/Abstract] OR digitalizacion[Title/Abstract] OR digitalizada[Title/Abstract] OR digitalizadas[Title/Abstract] OR digitalizaing[Title/Abstract] OR digitalization[Title/Abstract] OR digitalization'[Title/Abstract] OR digitalizations[Title/Abstract] OR digitalize[Title/Abstract] OR digitalized[Title/Abstract] OR digitalizer[Title/Abstract] OR digitalizers[Title/Abstract] OR digitalizes[Title/Abstract] OR digitalizing[Title/Abstract] OR digitalizzata[Title/Abstract] OR digitalizzazione[Title/Abstract] OR digitaljournal[Title/Abstract] OR digitalk[Title/Abstract] OR digitall[Title/Abstract] OR digitallis[Title/Abstract] OR digitallung[Title/Abstract] OR

digitally[Title/Abstract] OR digitally'[Title/Abstract] OR digitallyconnected[Title/Abstract] OR digitalmammography[Title/Abstract] OR digitalme[Title/Abstract] OR digitalmed[Title/Abstract] OR digitalmetrade[Title/Abstract] OR digitalmicrograph[Title/Abstract] OR digitalmicrographtrade[Title/Abstract] OR digitalmill[Title/Abstract] OR digitalmlpa[Title/Abstract] OR digitalo[Title/Abstract] OR digitaloid[Title/Abstract] OR digitaloides[Title/Abstract] OR digitaloidites[Title/Abstract] OR digitaloids[Title/Abstract] OR digitalonin[Title/Abstract] OR digitalopyranoside[Title/Abstract] OR digitalopyranosy[[Title/Abstract] OR digitalose[Title/Abstract] OR digitalosid[Title/Abstract] OR digitaloside[Title/Abstract] OR digitalosyl[Title/Abstract] OR digitalotherapy[Title/Abstract] OR digitalpcr[Title/Abstract] OR digitalpt[Title/Abstract] OR digitalradiography[Title/Abstract] OR digitalrectal[Title/Abstract] OR digitalrom[Title/Abstract] OR digitals[Title/Abstract] OR digitalslide[Title/Abstract] OR digitalslidearchive[Title/Abstract] OR digitalspiders[Title/Abstract] OR digitaltf[Title/Abstract] OR digitaltrade[Title/Abstract] OR digitalvhi[Title/Abstract] OR digitalyzed[Title/Abstract]) OR (technolog[Title/Abstract] OR technologeous[Title/Abstract] OR technologia[Title/Abstract] OR technologiae[Title/Abstract] OR technologic[Title/Abstract] OR technological[Title/Abstract] OR technological'[Title/Abstract] OR technologicalchallenge[Title/Abstract] OR technologicalforecasting[Title/Abstract] OR

technologicalforesight[Title/Abstract] OR technologicalization[Title/Abstract] OR technologically[Title/Abstract] OR technologically'[Title/Abstract] OR technologicalperspective[Title/Abstract] OR technologics[Title/Abstract] OR technologicus[Title/Abstract] OR technologie[Title/Abstract] OR technologie'[Title/Abstract] OR technologiebewertung[Title/Abstract] OR technologiees[Title/Abstract] OR technologielaan[Title/Abstract] OR technologien[Title/Abstract] OR technologiepark[Title/Abstract] OR technologies[Title/Abstract] OR technologies'[Title/Abstract] OR technologies's[Title/Abstract] OR technologiesare[Title/Abstract] OR technologiesartificial[Title/Abstract] OR technologiesguidance[Title/Abstract] OR technologiesis[Title/Abstract] OR technologiesrome[Title/Abstract] OR technologiessuch[Title/Abstract] OR technologiestm[Title/Abstract] OR technologiestrade[Title/Abstract] OR technologieswe[Title/Abstract] OR technologii[Title/Abstract] OR technologique[Title/Abstract] OR technologiques[Title/Abstract] OR technologis[Title/Abstract] OR technologisation[Title/Abstract] OR technologische[Title/Abstract] OR technologised[Title/Abstract] OR technologising[Title/Abstract] OR technologism[Title/Abstract] OR technologist[Title/Abstract] OR technologist'[Title/Abstract] OR technologist's[Title/Abstract] OR technologists[Title/Abstract] OR technologists'[Title/Abstract] OR technologists'attitudes[Title/Abstract] OR technologists's[Title/Abstract] OR technologization[Title/Abstract] OR technologize[Title/Abstract] OR technologized[Title/Abstract] OR

technologizing[Title/Abstract] OR technologlans[Title/Abstract] OR technologly[Title/Abstract] OR technology[Title/Abstract] OR technology'[Title/Abstract] OR technology"[Title/Abstract] OR technology's[Title/Abstract] OR technology1[Title/Abstract] OR technology16[Title/Abstract] OR technology3[Title/Abstract] OR technologyallows[Title/Abstract] OR technologyand[Title/Abstract] OR technologyassisted[Title/Abstract] OR technologyconsider[Title/Abstract] OR technologydagger[Title/Abstract] OR technologyevaluation[Title/Abstract] OR technologyfor[Title/Abstract] OR technologyhave[Title/Abstract] OR technologyhose[Title/Abstract] OR technologyin[Title/Abstract] OR technologyis[Title/Abstract] OR technologyit[Title/Abstract] OR technologymc[Title/Abstract] OR technologypreclinical[Title/Abstract] OR technologys[Title/Abstract] OR technologythat[Title/Abstract] OR technologyto[Title/Abstract] OR technologytrade[Title/Abstract] OR technologytranslation[Title/Abstract] OR technologyuser[Title/Abstract] OR technologyvarious[Title/Abstract])) OR (sensor[Title/Abstract] OR sensor'[Title/Abstract] OR sensor's[Title/Abstract] OR sensor1[Title/Abstract] OR sensor4pri[Title/Abstract] OR sensora[Title/Abstract] OR sensorad[Title/Abstract] OR sensoraid[Title/Abstract] OR sensoral[Title/Abstract] OR 
sensorale[Title/Abstract] OR sensorally[Title/Abstract] OR sensorand[Title/Abstract] OR sensorant[Title/Abstract] OR sensorapertures[Title/Abstract] OR sensorarray[Title/Abstract] OR sensorart[Title/Abstract] OR sensorband[Title/Abstract] OR sensorbility[Title/Abstract] OR sensorbod[Title/Abstract] OR sensorbox[Title/Abstract] OR sensorcaine[Title/Abstract] OR sensorcatheter[Title/Abstract] OR sensorchip[Title/Abstract] OR sensorchips[Title/Abstract] OR sensorcm[Title/Abstract] OR sensorconsists[Title/Abstract] OR sensordata[Title/Abstract] OR sensordb[Title/Abstract] OR sensordish[Title/Abstract] OR sensore[Title/Abstract] OR sensoready[Title/Abstract] OR sensorealization[Title/Abstract] OR sensored[Title/Abstract] OR sensoredge[Title/Abstract] OR sensoregulator[Title/Abstract] OR sensoremoval[Title/Abstract] OR sensoremoval'[Title/Abstract] OR sensores[Title/Abstract] OR sensorevery[Title/Abstract] OR sensoreverywhere[Title/Abstract] OR sensorex[Title/Abstract] OR sensorexhibited[Title/Abstract] OR sensorfor[Title/Abstract] OR sensorfret[Title/Abstract] OR sensorg[Title/Abstract] OR sensorgraft[Title/Abstract] OR sensorgram[Title/Abstract] OR sensorgrams[Title/Abstract] OR sensorgraphy[Title/Abstract] OR sensorhis[Title/Abstract] OR sensori[Title/Abstract] OR sensoria[Title/Abstract] OR sensoriactuators[Title/Abstract] OR sensoriais[Title/Abstract] OR sensorial[Title/Abstract] OR sensorial'[Title/Abstract] OR sensoriales[Title/Abstract] OR sensorialist[Title/Abstract] OR sensorialite[Title/Abstract] OR sensoriality[Title/Abstract] OR sensorialized[Title/Abstract] OR sensorially[Title/Abstract] OR sensorials[Title/Abstract] OR sensorialy[Title/Abstract] OR sensoribehavioural[Title/Abstract] OR sensoric[Title/Abstract] OR sensorica[Title/Abstract] OR sensorical[Title/Abstract] OR sensorically[Title/Abstract] OR sensoricortical[Title/Abstract] OR sensoricphysiological[Title/Abstract] OR sensorics[Title/Abstract] OR

sensoridiscriminative[Title/Abstract] OR sensoriel[Title/Abstract] OR sensorielle[Title/Abstract] OR sensorielles[Title/Abstract] OR sensoriels[Title/Abstract] OR sensories[Title/Abstract] OR sensorif[Title/Abstract] OR sensorigenesis[Title/Abstract] OR sensorii[Title/Abstract] OR sensoril[Title/Abstract] OR sensorilimbic[Title/Abstract] OR sensorily[Title/Abstract] OR sensorimetry[Title/Abstract] OR sensorimortor[Title/Abstract] OR sensorimoter[Title/Abstract] OR sensorimoteur[Title/Abstract] OR sensorimoteurs[Title/Abstract] OR sensorimotility[Title/Abstract] OR sensorimotor[Title/Abstract] OR

sensorimotor'[Title/Abstract] OR sensorimotoraffective[Title/Abstract] OR sensorimotoraxonal[Title/Abstract] OR sensorimotorcontrol[Title/Abstract] OR sensorimotorcortical[Title/Abstract] OR sensorimotori[Title/Abstract] OR sensorimotorial[Title/Abstract] OR sensorimotoric[Title/Abstract] OR sensorimotorrhythm[Title/Abstract] OR sensorimotorsystem[Title/Abstract] OR sensorimotory[Title/Abstract] OR sensorimototor[Title/Abstract] OR sensorimotrice[Title/Abstract] OR sensorimotrices[Title/Abstract] OR sensorimotricity[Title/Abstract] OR sensorin[Title/Abstract] OR sensorincludes[Title/Abstract] OR sensorineal[Title/Abstract] OR sensorineral[Title/Abstract] OR sensorineraul[Title/Abstract] OR sensorinerual[Title/Abstract] OR sensorinerural[Title/Abstract] OR sensoriness[Title/Abstract] OR sensorineu[Title/Abstract] OR sensorineual[Title/Abstract] OR sensorineueal[Title/Abstract] OR sensorineumral[Title/Abstract] OR sensorineur[Title/Abstract] OR sensorineura[Title/Abstract] OR sensorineural[Title/Abstract] OR sensorineural'[Title/Abstract] OR sensorineuralhearing[Title/Abstract] OR sensorineuralis[Title/Abstract] OR sensorineurally[Title/Abstract] OR sensorineurals[Title/Abstract] OR sensorineurepithelium[Title/Abstract] OR sensorineurinal[Title/Abstract] OR sensorineurocognitive[Title/Abstract] OR sensorineuroepithelium[Title/Abstract] OR sensorineurological[Title/Abstract] OR sensorineuronal[Title/Abstract] OR sensorineutral[Title/Abstract] OR sensoring[Title/Abstract] OR sensoringeural[Title/Abstract] OR sensorintegrative[Title/Abstract] OR sensorinueral[Title/Abstract] OR sensorinural[Title/Abstract] OR sensorio[Title/Abstract] OR sensorioculomotor[Title/Abstract] OR sensoriomotor[Title/Abstract] OR sensoriomotora[Title/Abstract] OR sensoriomotoras[Title/Abstract] OR sensoriomotriz[Title/Abstract] OR sensorion[Title/Abstract] OR sensorioneural[Title/Abstract] OR sensoriperception[Title/Abstract] OR sensoriperceptual[Title/Abstract] OR sensoriphasic[Title/Abstract] OR sensoriphobia[Title/Abstract] OR sensoriprocessing[Title/Abstract] OR sensoris[Title/Abstract] OR sensorisation[Title/Abstract] OR sensorische[Title/Abstract] OR sensorised[Title/Abstract] OR sensorisensory[Title/Abstract] OR sensoriske[Title/Abstract] OR sensorisomatic[Title/Abstract] OR sensoristasis[Title/Abstract] OR sensoristatic[Title/Abstract] OR sensoristic[Title/Abstract] OR sensoristics[Title/Abstract] OR sensoristrain[Title/Abstract] OR sensoristrain'[Title/Abstract] OR sensorithm[Title/Abstract] OR sensoritmotor[Title/Abstract] OR sensoritopic[Title/Abstract] OR sensoritrigeminal[Title/Abstract] OR sensoritrophic[Title/Abstract] OR sensority[Title/Abstract] OR sensorium[Title/Abstract] OR sensorium'[Title/Abstract] OR sensoriums[Title/Abstract] OR sensorius[Title/Abstract] OR sensorivagal[Title/Abstract] OR sensorization[Title/Abstract] OR sensorize[Title/Abstract] OR sensorized[Title/Abstract] OR sensorized'[Title/Abstract] OR sensorizing[Title/Abstract] OR sensorless[Title/Abstract] OR sensorlike[Title/Abstract] OR sensorlink[Title/Abstract] OR sensorlog[Title/Abstract] OR sensormat[Title/Abstract] OR sensormedic[Title/Abstract] OR sensormedics[Title/Abstract] OR 
sensormedicus[Title/Abstract] OR sensormedix[Title/Abstract] OR sensorml[Title/Abstract] OR sensormotor[Title/Abstract] OR sensornet[Title/Abstract] OR sensornetworks[Title/Abstract] OR sensorneural[Title/Abstract] OR sensornodes[Title/Abstract] OR sensornye[Title/Abstract] OR sensoro[Title/Abstract] OR sensorograms[Title/Abstract] OR sensoromotor[Title/Abstract] OR sensoromotoric[Title/Abstract] OR sensoroneural[Title/Abstract] OR sensoror[Title/Abstract] OR sensorotoxin[Title/Abstract] OR sensorpoly[Title/Abstract] OR sensorproperties[Title/Abstract] OR sensorregulator[Title/Abstract] OR sensorreporters[Title/Abstract] OR sensorresponse[Title/Abstract] OR sensors[Title/Abstract] OR sensors'[Title/Abstract] OR sensors's[Title/Abstract] OR sensors2016[Title/Abstract] OR sensorsa[Title/Abstract] OR sensorsand[Title/Abstract] OR sensorsbased[Title/Abstract] OR sensorscope[Title/Abstract] OR sensorselection[Title/Abstract] OR sensorsetting[Title/Abstract] OR sensorship[Title/Abstract] OR sensorshowed[Title/Abstract] OR sensorsi[Title/Abstract] OR sensorsis[Title/Abstract] OR sensorspace[Title/Abstract] OR sensorsthe[Title/Abstract] OR sensorstrips[Title/Abstract] OR sensorsuse[Title/Abstract] OR sensorsv[Title/Abstract] OR sensorswere[Title/Abstract] OR sensort[Title/Abstract] OR sensortag[Title/Abstract] OR sensortalk[Title/Abstract] OR

sensortech[Title/Abstract] OR sensortechnik[Title/Abstract] OR sensortechnology[Title/Abstract] OR sensortek[Title/Abstract] OR sensorthat[Title/Abstract] OR sensorthings[Title/Abstract] OR sensorto[Title/Abstract] OR sensortouch[Title/Abstract] OR sensortrade[Title/Abstract] OR sensorts[Title/Abstract] OR sensorwas[Title/Abstract] OR sensorweb[Title/Abstract] OR sensory[Title/Abstract] OR sensory'[Title/Abstract] OR sensory's[Title/Abstract] OR sensoryanalysis[Title/Abstract] OR sensorydeprivation[Title/Abstract] OR sensoryevoked[Title/Abstract] OR sensorygc[Title/Abstract] OR sensoryical[Title/Abstract] OR sensoryinteractions[Title/Abstract] OR sensorylike[Title/Abstract] OR sensorymodality[Title/Abstract] OR sensorymotor[Title/Abstract] OR sensorynav1[Title/Abstract] OR sensoryneural[Title/Abstract] OR sensoryneuropathy[Title/Abstract] OR sensoryomics[Title/Abstract] OR sensorypredominant[Title/Abstract] OR sensoryprocessing[Title/Abstract] OR sensoryrecovery[Title/Abstract] OR sensoryrelated[Title/Abstract] OR sensoryrhodopsin[Title/Abstract] OR sensoryrizotomy[Title/Abstract] OR sensorytransduction[Title/Abstract] OR sensorytreat[Title/Abstract] OR sensorytreat's[Title/Abstract])) OR (robot[Title/Abstract] OR robot'[Title/Abstract] OR robot'consists[Title/Abstract] OR robot'electronically[Title/Abstract] OR robot's[Title/Abstract] OR robota[Title/Abstract] OR robotac[Title/Abstract] OR robotanalyst[Title/Abstract] OR robotanalyst's[Title/Abstract] OR robotarium[Title/Abstract] OR robotassisted[Title/Abstract] OR robotassistedlaparoscopic[Title/Abstract] OR robotcar[Title/Abstract] OR robotechnologies[Title/Abstract] OR robotechnologist[Title/Abstract] OR roboteeg[Title/Abstract] OR roboter[Title/Abstract] OR roboterassistiert[Title/Abstract] OR roboterassistierte[Title/Abstract] OR roboterassistierter[Title/Abstract] OR robotfoto[Title/Abstract] OR robotham[Title/Abstract] OR robotherapist[Title/Abstract] OR robotherapist'[Title/Abstract] OR robotherapy[Title/Abstract] OR robothics[Title/Abstract] OR roboti[Title/Abstract] OR robotic[Title/Abstract] OR robotic'[Title/Abstract] OR robotica[Title/Abstract] OR robotical[Title/Abstract] OR robotically[Title/Abstract] OR roboticallyassisted[Title/Abstract] OR roboticamente[Title/Abstract] OR roboticapproach[Title/Abstract] OR roboticarm[Title/Abstract] OR roboticas[Title/Abstract] OR roboticassisted[Title/Abstract] OR roboticbed[Title/Abstract] OR roboticbird[Title/Abstract] OR roboticians[Title/Abstract] OR roboticist[Title/Abstract] OR roboticists[Title/Abstract] OR roboticists'[Title/Abstract] OR roboticization[Title/Abstract] OR roboticized[Title/Abstract] OR roboticizes[Title/Abstract] OR robotico[Title/Abstract] OR roboticos[Title/Abstract] OR roboticpancreatoduodenectomy[Title/Abstract] OR robotics[Title/Abstract] OR robotics'[Title/Abstract] OR roboticslab[Title/Abstract] OR roboticsurgery[Title/Abstract] OR roboticsystem[Title/Abstract] OR robotik[Title/Abstract] OR robotiker[Title/Abstract] OR robotiq[Title/Abstract] OR robotique[Title/Abstract] OR robotiquette[Title/Abstract] OR robotiquette'[Title/Abstract] OR robotisation[Title/Abstract] OR robotisches[Title/Abstract] OR robotise[Title/Abstract] OR robotised[Title/Abstract] OR robotisee[Title/Abstract] OR robotitian[Title/Abstract] OR robotix[Title/Abstract] OR robotization[Title/Abstract] OR robotize[Title/Abstract] OR robotized[Title/Abstract] OR robotizing[Title/Abstract] OR robotizzato[Title/Abstract] OR robotless[Title/Abstract] OR robotlike[Title/Abstract] OR robotmajsebeszet[Title/Abstract] OR robotmanipulators[Title/Abstract] OR robotmediated[Title/Abstract] OR robotnikinin[Title/Abstract] OR robotnon[Title/Abstract] OR roboto[Title/Abstract] OR robotocs[Title/Abstract] OR robotok[Title/Abstract] OR robotol[Title/Abstract] OR robototherapy[Title/Abstract] OR robotrac[Title/Abstract] OR robotrat[Title/Abstract] OR robotreviewer[Title/Abstract] OR robotreviewer's[Title/Abstract] OR robotripping[Title/Abstract] OR robotrobot[Title/Abstract] OR robotron[Title/Abstract] OR robots[Title/Abstract] OR robots'[Title/Abstract] OR robots'designs[Title/Abstract] OR robotsci[Title/Abstract] OR robotscientist[Title/Abstract] OR robotsebeszet[Title/Abstract] OR robotsebeszeti[Title/Abstract] OR robotsfor[Title/Abstract] OR robotti[Title/Abstract] OR robottom[Title/Abstract] OR robottrade[Title/Abstract] OR robotuna[Title/Abstract] OR 
robotutor[Title/Abstract] OR robotworld[Title/Abstract])) OR internet[Title/Abstract]) OR social media[Title/Abstract]) OR (phone[Title/Abstract] OR phone'[Title/Abstract] OR phone's[Title/Abstract] OR phonear[Title/Abstract] OR phonebased[Title/Abstract] OR phonebook[Title/Abstract] OR

phoneburst[Title/Abstract] OR phonecall[Title/Abstract] OR phonecard[Title/Abstract] OR phonecardiographic[Title/Abstract] OR phonecians[Title/Abstract] OR phonecians'[Title/Abstract] OR phonectically[Title/Abstract] OR phoned[Title/Abstract] OR phonedependency[Title/Abstract] OR phoneeded[Title/Abstract] OR phoneeutria[Title/Abstract] OR phonefriend[Title/Abstract] OR phonegap[Title/Abstract] OR phoneigen[Title/Abstract] OR phonein[Title/Abstract] OR phoneix[Title/Abstract] OR phonelectrocardiography[Title/Abstract] OR phoneline[Title/Abstract] OR phonem[Title/Abstract] OR phonema[Title/Abstract] OR phonemas[Title/Abstract] OR phonematic[Title/Abstract] OR phonematically[Title/Abstract] OR phonematics[Title/Abstract] OR phonematique[Title/Abstract] OR phonemchanograms[Title/Abstract] OR phoneme[Title/Abstract] OR phoneme'[Title/Abstract] OR phoneme's[Title/Abstract] OR phonemegrapheme[Title/Abstract] OR phonemena[Title/Abstract] OR phonemes[Title/Abstract] OR phonemes'[Title/Abstract] OR phonemeter[Title/Abstract] OR phonemethod[Title/Abstract] OR phonemic[Title/Abstract] OR phonemicably[Title/Abstract] OR phonemically[Title/Abstract] OR phonemicisation[Title/Abstract] OR phonemicization[Title/Abstract] OR phonemics[Title/Abstract] OR phonemisation[Title/Abstract] OR phonemoformation[Title/Abstract] OR phonemonon[Title/Abstract] OR phonemotopic[Title/Abstract] OR phonems[Title/Abstract] OR phonendoscope[Title/Abstract] OR phonendoscopes[Title/Abstract] OR phonendoscopic[Title/Abstract] OR phonenix[Title/Abstract] OR phonenterographia[Title/Abstract] OR phoneomic[Title/Abstract] OR phonequant[Title/Abstract] OR phonequit[Title/Abstract] OR

phonereporting[Title/Abstract] OR phonernically[Title/Abstract] OR phonerpeton[Title/Abstract] OR phones[Title/Abstract] OR phones'[Title/Abstract] OR phones4u[Title/Abstract] OR phoneshop[Title/Abstract] OR phoneside[Title/Abstract] OR phonesimulated[Title/Abstract] OR phonesis[Title/Abstract] OR phonesoap[Title/Abstract] OR phonesonhuman[Title/Abstract] OR phonesthemes[Title/Abstract] OR phonesthemic[Title/Abstract] OR phonet[Title/Abstract] OR phonethep[Title/Abstract] OR phonetic[Title/Abstract] OR phonetica[Title/Abstract] OR phonetical[Title/Abstract] OR phonetically[Title/Abstract] OR phoneticeffects[Title/Abstract] OR phonetician[Title/Abstract] OR phonetician's[Title/Abstract] OR phoneticians[Title/Abstract] OR phoneticization[Title/Abstract] OR phoneticized[Title/Abstract] OR phonetics[Title/Abstract] OR phonetics'[Title/Abstract] OR phonetik[Title/Abstract] OR phonetion[Title/Abstract] OR phonetique[Title/Abstract] OR phonetisation[Title/Abstract] OR phonetogram[Title/Abstract] OR phonetograms[Title/Abstract] OR phonetograph[Title/Abstract] OR phonetographic[Title/Abstract] OR phonetography[Title/Abstract] OR phonetometric[Title/Abstract] OR

phonetoxin[Title/Abstract] OR phonetype[Title/Abstract] OR phonetypical[Title/Abstract] OR phoneus[Title/Abstract] OR phoneuse[Title/Abstract] OR phoneutria[Title/Abstract] OR phoneutriatoxin[Title/Abstract] OR phoneutrism[Title/Abstract] OR phoney[Title/Abstract] OR phoneyusa[Title/Abstract])) OR (telephone[Title/Abstract] OR telephone'[Title/Abstract] OR telephone's[Title/Abstract] OR telephoneassisted[Title/Abstract] OR telephonecare[Title/Abstract] OR telephoned[Title/Abstract] OR telephoneear[Title/Abstract] OR telephoneinterviews[Title/Abstract] OR telephoners[Title/Abstract] OR telephoners'[Title/Abstract] OR telephones[Title/Abstract] OR telephonesex[Title/Abstract] OR telephonetics[Title/Abstract])) OR online[Title/Abstract]) OR (ipad[Title/Abstract] OR ipad's[Title/Abstract] OR ipad120[Title/Abstract] OR ipad2[Title/Abstract] OR ipad20[Title/Abstract] OR ipad2s[Title/Abstract] OR ipad4[Title/Abstract] OR ipada[Title/Abstract] OR ipadair[Title/Abstract] OR ipadam[Title/Abstract] OR ipadar[Title/Abstract] OR ipadb[Title/Abstract] OR ipade[Title/Abstract] OR ipadech[Title/Abstract] OR ipadh[Title/Abstract] OR ipadia[Title/Abstract] OR ipadm[Title/Abstract] OR ipadnych[Title/Abstract] OR ipado[Title/Abstract] OR ipados[Title/Abstract] OR ipads[Title/Abstract] OR ipadstrade[Title/Abstract] OR ipadt[Title/Abstract] OR ipadtrade[Title/Abstract] OR ipadu[Title/Abstract] OR ipadur1[Title/Abstract] OR ipadvas[Title/Abstract])) OR (computer[Title/Abstract] OR computer'[Title/Abstract] OR computer's[Title/Abstract] OR computer18[Title/Abstract] OR computer19[Title/Abstract] OR computer3[Title/Abstract] OR computeradapted[Title/Abstract] OR computeraided[Title/Abstract] OR computerally[Title/Abstract] OR computeranalysis[Title/Abstract] OR computerand[Title/Abstract] OR computerangiography[Title/Abstract] OR computeranimated[Title/Abstract] OR computerarthrometry[Title/Abstract] OR computerassisted[Title/Abstract] OR computerassistierte[Title/Abstract] OR computerbase[Title/Abstract] OR computerbased[Title/Abstract] OR computercalculated[Title/Abstract] OR computerchemie[Title/Abstract] OR computerclassified[Title/Abstract] OR computercode[Title/Abstract] OR computercontrolled[Title/Abstract] OR computerd[Title/Abstract] OR computerdesigned[Title/Abstract] OR computerdocking[Title/Abstract] OR computerdokumentation[Title/Abstract] OR computerdriven[Title/Abstract] OR computered[Title/Abstract] OR computerenhanced[Title/Abstract] OR computerese[Title/Abstract] OR 
computereyes[Title/Abstract] OR computerezed[Title/Abstract] OR computerfiled[Title/Abstract] OR computerfitted[Title/Abstract] OR computergame[Title/Abstract] OR computergames[Title/Abstract] OR computergenerated[Title/Abstract] OR computergestutzte[Title/Abstract] OR computergestutzter[Title/Abstract] OR computergraphic[Title/Abstract] OR computergraphical[Title/Abstract] OR computergraphically[Title/Abstract] OR computergraphics[Title/Abstract] OR computergraphs[Title/Abstract] OR computerguided[Title/Abstract] OR computerheld[Title/Abstract] OR computeric[Title/Abstract] OR computerin[Title/Abstract] OR computering[Title/Abstract] OR computerintensive[Title/Abstract] OR computerinterpolated[Title/Abstract] OR computeris[Title/Abstract] OR computerisation[Title/Abstract] OR computerisations[Title/Abstract] OR computerise[Title/Abstract] OR computerised[Title/Abstract] OR computerised'[Title/Abstract] OR computerisedprovider[Title/Abstract] OR computerisee[Title/Abstract] OR computerises[Title/Abstract] OR computerising[Title/Abstract] OR computerism[Title/Abstract] OR computerizability[Title/Abstract] OR computerizable[Title/Abstract] OR computerizada[Title/Abstract] OR computerization[Title/Abstract] OR computerization'[Title/Abstract] OR computerization's[Title/Abstract] OR computerizd[Title/Abstract] OR computerize[Title/Abstract] OR computerizea[Title/Abstract] OR computerized[Title/Abstract] OR computerized'[Title/Abstract] OR computerizedcardiotocography[Title/Abstract] OR computerizeddynamic[Title/Abstract] OR computerizeds[Title/Abstract] OR computerizedt[Title/Abstract] OR computerizedtomographic[Title/Abstract] OR computerizes[Title/Abstract] OR computerizewd[Title/Abstract] OR computerizied[Title/Abstract] OR computerizing[Title/Abstract] OR computerizsed[Title/Abstract] OR computerizzata[Title/Abstract] OR computerlab[Title/Abstract] OR computerlabelled[Title/Abstract] OR computerland[Title/Abstract] OR computerless[Title/Abstract] OR computerlike[Title/Abstract] OR computerlink[Title/Abstract] OR computerlink's[Title/Abstract] OR computerly[Title/Abstract] OR computermediated[Title/Abstract] OR computermen[Title/Abstract] OR computermotion[Title/Abstract] OR computernavigated[Title/Abstract] OR computernavigation[Title/Abstract] OR computerneuron[Title/Abstract] OR computero[Title/Abstract] OR computerogenic[Title/Abstract] OR computerologist[Title/Abstract] OR computeromography[Title/Abstract] OR computeros[Title/Abstract] OR computerpart[Title/Abstract] OR computerphobe[Title/Abstract] OR computerphobia[Title/Abstract] OR computerphobia'[Title/Abstract] OR computerphobic[Title/Abstract] OR computerprogram[Title/Abstract] OR computerprogrammen[Title/Abstract] OR computerprogrammes[Title/Abstract] OR computerprograms[Title/Abstract] OR computerreadable[Title/Abstract] OR computerrelated[Title/Abstract] OR computerrhea[Title/Abstract] OR computerrised[Title/Abstract] OR computers[Title/Abstract] OR computers'[Title/Abstract] OR computers"[Title/Abstract] OR computerscan[Title/Abstract] OR computerscreen[Title/Abstract] OR computersimulation[Title/Abstract] OR computersimulations[Title/Abstract] OR computersmartphone[Title/Abstract] OR computersonography[Title/Abstract] OR computerspeak[Title/Abstract] OR computerspiel[Title/Abstract] OR computerspielabhangigkeit[Title/Abstract] OR computerspielsucht[Title/Abstract] OR computerstored[Title/Abstract] OR computersupported[Title/Abstract] OR computersystem[Title/Abstract] OR computersystems[Title/Abstract] OR computertime[Title/Abstract] OR computertized[Title/Abstract] OR computertomografie[Title/Abstract] OR computertomografischen[Title/Abstract] OR computertomogram[Title/Abstract] OR computertomogramm[Title/Abstract] OR computertomogramms[Title/Abstract] OR computertomograms[Title/Abstract] OR computertomograph[Title/Abstract] OR computertomographic[Title/Abstract] OR computertomographical[Title/Abstract] OR computertomographically[Title/Abstract] OR computertomographie[Title/Abstract] OR computertomographies[Title/Abstract] OR computertomographs[Title/Abstract] OR computertomography[Title/Abstract] OR computerunterstutzte[Title/Abstract] OR computerunterstutztes[Title/Abstract] OR computerusage[Title/Abstract] OR computerville[Title/Abstract] OR computervirus[Title/Abstract] OR computervision[Title/Abstract] OR computerworld[Title/Abstract])) OR (electronic[Title/Abstract] OR electronic'[Title/Abstract] OR electronic's[Title/Abstract] OR electronica[Title/Abstract] OR electronical[Title/Abstract] OR electronicall[Title/Abstract] OR electronically[Title/Abstract] OR electronically'[Title/Abstract] OR electronicallyexcited[Title/Abstract] OR electronicaly[Title/Abstract] OR electronicas[Title/Abstract] OR electronicbibliographic[Title/Abstract] OR electroniccards[Title/Abstract] OR electroniccase[Title/Abstract] OR electronicdatabase[Title/Abstract] OR electronicdocument[Title/Abstract] OR electronicfans[Title/Abstract] OR electroniclly[Title/Abstract] OR electronicmaterials[Title/Abstract] OR electronicmedical[Title/Abstract] OR electronicmedsman[Title/Abstract] OR electronicmessage[Title/Abstract] OR electronico[Title/Abstract] OR electronicos[Title/Abstract] OR electronicpatientrecords[Title/Abstract] OR electronicplatform[Title/Abstract] OR electronicquestionnaire[Title/Abstract] OR electronicreadout[Title/Abstract] OR electronicrecruitment[Title/Abstract] OR 
electronicrograph[Title/Abstract] OR electronics[Title/Abstract] OR electronics'[Title/Abstract] OR electronicsearch[Title/Abstract] OR electronicssensors[Title/Abstract] OR electronictemplate[Title/Abstract] OR electronicwaste[Title/Abstract] OR electronicwe[Title/Abstract])) OR web[Title/Abstract])) AND ((Clinical Trial[ptyp] OR Controlled Clinical Trial[ptyp] OR Journal Article[ptyp] OR Meta-Analysis[ptyp] OR Observational Study[ptyp] OR Randomized Controlled Trial[ptyp] OR Review[ptyp] OR systematic[sb]) AND ("2010/01/01"[PDAT] : "2019/07/31"[PDAT]) AND "humans"[MeSH Terms])

\section{EMBASE}

1. Ioneliness/

2. Loneliness.ti,ab.

3. (lonely or social isolation).ti,ab.

4. 1 or 2 or 3

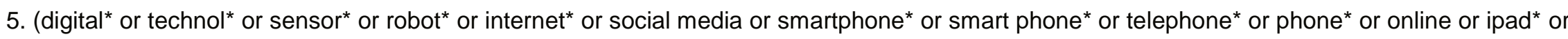
computer* or electronic* or Web).ti,ab.

6. robotics/

7. Internet/

8. social media/

9. smartphone/

10. personal digital assistant/

11. computer/

12. telephone/

13. 5 or 6 or 7 or 8 or 9 or 10 or 11 or 12

14. 4 and 13

15. limit 14 to (human and english language)

16. limit 15 to $y r=" 2010$-Current" 


\section{LONELINESS/}

2. Loneliness.ti,ab.

3. (lonely or social isolation).ti,ab.

4. 1 or 2 or 3

5. (digital ${ }^{*}$ or technol ${ }^{*}$ or sensor ${ }^{*}$ or robot ${ }^{*}$ or internet ${ }^{*}$ or social media or smartphone ${ }^{*}$ or smart phone* or telephone* or phone* or online or ipad ${ }^{*}$ or computer* or electronic* or Web).ti,ab.
6. ROBOTICS/

7. INTERNET/

8. Social Media/

9. TELEPHONE/

10. Smartphone/

11. Computers, Handheld/

12. COMPUTERS/

13.5 or 6 or 7 or 8 or 9 or 10 or 11 or 12

14. 4 and 13

15. limit 14 to (english language and humans and yr="2010 -Current" and (clinical trial, all or evaluation studies or journal article or meta analysis or observational study or "review" or systematic reviews)) 
Interface - EBSCOhost Research Databases

Search Screen - Advanced Search

Database - CINAHL

((MH loneliness) OR (TX loneliness) OR ((TX lonely) OR (TX social isolation))) AND ((TX (digital* OR technol* OR sensor* OR robot* OR social media OR smartphone* OR smart phone* OR phone* OR online OR ipad* OR computer ${ }^{*}$ OR elctronic* OR Web)) OR (MH technology) OR (MH robotics) OR (MH internet) OR (MH social media) OR (MH smartphone) OR (MH telephone) OR (MH computers, hand-held) OR (MH computers, portable) OR (MH world wide web))

\section{WEB OF SCIENCE}

TOPIC: (loneliness) OR TOPIC: (lonely) OR TOPIC: (social isolation)

Indexes=SCI-EXPANDED, SSCI, A\&HCI, ESCI Timespan=1900-2019

TOPIC: (digital) OR TOPIC: (technolog*) OR TOPIC: (sensor*) OR TOPIC: (robot*) OR TOPIC: (internet) OR TOPIC: (social

\#2 media) OR TOPIC: (smart phone*) OR TOPIC: (online) OR TOPIC: (ipad*) OR TOPIC: (computer*) OR TOPIC: (electronic*) OR TOPIC: (web) Indexes=SCI-EXPANDED, SSCI, A\&HCI, ESCI Timespan=1900-2019

\#2 AND \#1

Indexes=SCI-EXPANDED, SSCI, A\&HCI, ESCI Timespan=1900-2019

\#2 AND \#1

\# 4 Refined by: PUBLICATION YEARS: ( 2019 OR 2018 OR 2017 OR 2016 OR 2015 OR 2014 OR 2013 OR 2012 OR 2011 OR 2010 ) Indexes=SCI-EXPANDED, SSCI, A\&HCI, ESCI Timespan=1900-2019

\#2 AND \#1

Refined by: PUBLICATION YEARS: ( 2019 OR 2018 OR 2017 OR 2016 OR 2015 OR 2014 OR 2013 OR 2012 OR 2011 OR 2010 ) AND DOCUMENT TYPES: ( ARTICLE OR REVIEW )

Indexes=SCI-EXPANDED, SSCI, A\&HCI, ESCI Timespan=1900-2019

\#2 AND \#1

\# 6 Refined by: PUBLICATION YEARS: ( 2019 OR 2018 OR 2017 OR 2016 OR 2015 OR 2014 OR 2013 OR 2012 OR 2011 OR 2010 ) AND DOCUMENT TYPES: ( ARTICLE OR REVIEW ) AND LANGUAGES: ( ENGLISH )

Indexes=SCI-EXPANDED, SSCI, A\&HCI, ESCI Timespan=1900-2019

Database: Web of Science Core Collection. 